\title{
OPTIMAL TRANSACTION FILTERS UNDER TRANSITORY TRADING OPPORTUNITIES: Theory and Empirical Illustration
}

RONALD J. BALVERS

West Virginia University, rbalvers@wvu.edu

YANGRUWU

Rutgers University, yangruwu@andromeda.rutgers.edu

Follow this and additional works at: https://fordham.bepress.com/crif_seminar_series

Part of the Finance and Financial Management Commons

\section{Recommended Citation}

BALVERS, RONALD J. and WU, YANGRU, "OPTIMAL TRANSACTION FILTERS UNDER TRANSITORY TRADING OPPORTUNITIES: Theory and Empirical Illustration" (2003). CRIF Seminar series. 21.

https://fordham.bepress.com/crif_seminar_series/21 


\title{
OPTIMAL TRANSACTION FILTERS UNDER TRANSITORY TRADING OPPORTUNITIES:

\author{
Theory and Empirical Illustration*
}

\author{
RONALD J. BALVERS \\ Division of Economics and Finance \\ College of Business \\ West Virginia University \\ Morgantown, WV 26506 \\ rbalvers@wvu.edu \\ Phone: (304) 293-7880 \\ Fax: (304) 293-2233
}

\author{
YANGRU WU \\ Department of Finance \\ Rutgers Business School-Newark \& New Brunswick \\ Rutgers University \\ Newark, NJ 07102-1820 \\ yangruwu@andromeda.rutgers.edu \\ Phone: (973) 353-1146 \\ Fax: (973) 353-1233
}

\section{December 2003}

\begin{abstract}
If transitory profitable trading opportunities exist, filter rules are used in practice to mitigate transaction costs. The filter size is difficult to determine a priori. Our paper uses a dynamic programming framework to design a filter that is optimal in the sense of maximizing expected returns after transaction costs. The optimal filter size depends crucially on the degree of persistence of the profitable trading opportunities, on transaction cost, and on the standard deviation of shocks.
\end{abstract}

We apply our theoretical results to foreign exchange trading by parameterizing the moving average strategy often employed in foreign exchange markets. The parameterization implies the same decisions as the moving average rule, in the absence of transaction costs, but has the advantage of translating the buy/sell signal into the same units as the transaction costs so that the optimal filter can be calculated.

Application to daily dollar-yen trading demonstrates that the optimal filter can differ dramatically from a naï ve filter equal to the transaction cost. We confirm that daily moving average foreign exchange trading generates positive returns that disappear after accounting for transaction costs. However, when the optimal filter is used, returns after transaction costs remain positive and are higher than for naï ve filters.

Keywords: Transaction Costs, Filter Rules, Trading Strategies, Foreign Exchange

\footnotetext{
* We would like to thank Gershon Alperovich, Ake Blomqvist, Justin Chan, Chong Tze Chua, Aditya Goenka, Dong Hong and seminar participants at the National University of Singapore, the University of Hong Kong and Singapore Management University for helpful comments. Yangru Wu would like to thank the Research Council of Rutgers University and Rutgers Business School for financial support. Part of this work was completed while Yangru Wu visited the Hong Kong Institute for Monetary Research. He thanks the Institutes for its hospitality.
} 


\title{
OPTIMAL TRANSACTION FILTERS UNDER TRANSITORY TRADING OPPORTUNITIES: \\ Theory and Empirical Illustration
}

\begin{abstract}
If transitory profitable trading opportunities exist, filter rules are used in practice to mitigate transaction costs. The filter size is difficult to determine a priori. Our paper uses a dynamic programming framework to design a filter that is optimal in the sense of maximizing expected returns after transaction costs. The optimal filter size depends crucially on the degree of persistence of the profitable trading opportunities, on transaction cost, and on the standard deviation of shocks.

We apply our theoretical results to foreign exchange trading by parameterizing the moving average strategy often employed in foreign exchange markets. The parameterization implies the same decisions as the moving average rule, in the absence of transaction costs, but has the advantage of translating the buy/sell signal into the same units as the transaction costs so that the optimal filter can be calculated.

Application to daily dollar-yen trading demonstrates that the optimal filter can differ dramatically from a naï ve filter equal to the transaction cost. We confirm that daily moving average foreign exchange trading generates positive returns that disappear after accounting for transaction costs. However, when the optimal filter is used, returns after transaction costs remain positive and are higher than for naï ve filters.
\end{abstract}

Keywords: Transaction Costs, Filter Rules, Trading Strategies, Foreign Exchange 


\section{Introduction}

It is inarguable that opportunities for above-normal returns are available to market participants at some level. These opportunities may be exploitable for instance at an intra-daily frequency as a reward for information acquisition when markets are efficient, or at a lower frequency, to market timers when markets are inefficient. By nature these profit opportunities are predicable but transitory, and transaction costs may be a major impediment in exploiting them. ${ }^{1}$ This paper explores the optimal trading strategy when transitory opportunities exist and transactions are costly.

The model we present is applicable to the arbitraging of microstructure inefficiencies that require frequent and timely transactions, which may be largely riskless. An example is uncovered interest speculation where a trader takes either one side of the market or the reverse. Alternatively, a trader arbitrages differences between a stock's return and that of one of its derivatives: going long on the arbitrage position or reversing the position and going short. The model also applies to momentum or mean reversion trading when these phenomena may reflect inefficiencies due to cognitive biases.

Starting with Fama and Blume (1966), trading rules often involve a "filter" that prompts one to trade only if a realization exceeds a benchmark by a certain percentage. For instance in foreign exchange trading an $\mathrm{x} \%$ filter might stipulate that a currency be purchased if its current exchange value exceeds some moving average of its past values by more than $\mathrm{x} \%$. Presumably, such a filter reduces trading and thus transaction costs. The idea is that if the trade indicator is "weak" the expected return from the transaction

\footnotetext{
${ }^{1}$ For instance, Grundy and Martin (2001) express doubt that the anomalous momentum profits survive transaction costs, and Hanna and Ready (2001) find that the momentum profits are substantially reduced when transactions costs are accounted for. Lesmond, Schill, and Zhou (2003) conclude more strongly that momentum profits with transactions costs are illusory.
} 
may not compensate for the transaction cost. Knez and Ready (1996) and Cooper (1999), for instance, explore different filters and find that the after-transaction-cost returns improve compared to trading strategies with zero filter. The problem with the filter approach is that there is no way of knowing a priori which percentage filter would be reasonable, since the buy/sell signal and the transaction cost are not in the same units. This also implies that there is no discipline against data mining for researchers: a large number of filters can be tried until the strategy generates positive net returns.

The purpose of this paper is to design an optimal filter that maximizes the expected return net of transaction cost. To accomplish this we employ a "parametric" approach (see for instance Balvers, Wu, and Gilliland, 2000) that allows the trading signal and the transaction cost to be in the same units. The optimal filter depends on the exact balance between maintaining the most profitable transactions and minimizing the transactions costs.

The optimal filter can be no larger than the transaction cost (plus interest). This is clear because there is no reason to exclude trades that have an immediate expected return larger than the transaction cost. In general the optimal filter is significantly smaller than the transaction cost. This occurs when the expected return is persistent: even if the immediate return from switching is less than the transaction cost, the persistence of the expected return makes it likely that an additional return is foregone in future periods by not switching. Roughly, the filter is given by the transaction cost times a factor related to the probability that a switch occurs. Our model characterizes the determinants of the filter in general and provides an exact solution for the filter under the assumption of changes in expected returns being uniformly distributed. In exploring the effect of transaction costs when returns are predictable, this paper follows Balduzzi 
and Lynch (1999) and Lynch and Balduzzi (2000). ${ }^{2}$ Their focus, however, differs significantly from ours in that they consider the utility effects and portfolio rebalancing decisions, respectively, in a life cycle portfolio choice framework. They simulate the welfare cost and portfolio rebalancing decisions given a trader's constant relative risk aversion utility function, but they do not provide analytical solutions and it is difficult to use their approach to quantify the optimal trading strategies for particular applications. Our approach, in contrast, provides specific theoretical results yielding insights into the factors affecting optimal trading strategies. Moreover our results can be applied based on observable market characteristics that do not depend on subjective utility function specifications.

In contrast to Balduzzi and Lynch (1999) and Lynch and Balduzzi (2000), we abstract from risk considerations. This simplifies our analysis considerably and is reasonable in a variety of circumstances. First, we can think of the raw returns as market-risk-adjusted returns, with whichever risk model is considered appropriate. The market risk adjustment is sufficient to account for all risk as long as trading occurs at the margins of an otherwise well-diversified portfolio. Second, in particular at intra-daily frequencies, traders may create arbitrage positions so that risk is irrelevant. Third, in many cases risk considerations are secondary compared to the gains in expected return.

Our framework implies that trading strategy returns using the optimal filter should be smaller than the returns when transaction costs are ignored but larger than for any other filter strategy with transaction costs, in particular those for which the filter is zero percent or equal to the transaction cost. We apply our optimal filter to a natural case for our model: daily foreign exchange trading in the yen/dollar market. As is

\footnotetext{
${ }^{2}$ There is a far more extensive literature considering investment choices under transaction costs when returns are not predictable. See Liu and Loewenstein (2002) for a recent example.
} 
well-known [see for instance Sweeney (1986), LeBaron (1998), and Qi and Wu (2002)], simple movingaverage trading rules generate positive expected returns (with or without risk adjustment) in the foreign exchange market. However, for daily trading, returns net of transaction costs are negative if no filter is applied.

We find that for the optimal filter the net returns are still significantly positive and higher than that when the filter is set equal to the transaction cost. Furthermore, the optimal filter derived from the theory given a uniform distribution and two optimal filters derived numerically under normality and bootstrapping assumptions all generate similar results that are moreover relatively close to the ex post maximizing filter for actual data. These results are important as they suggest an approach for employing trading strategies with filters to deal with transactions cost, without leading to excessive data mining. The results also hint that in some cases conclusions of abnormal profits disappearing after accounting for transaction costs may be worth revisiting.

The next section develops the theoretical model and provides a general characterization of the optimal filter for an ARMA(1,1) returns process with general shocks, as well as a specific formula for the case when the shocks follow the uniform distribution. In section II, we apply the model to uncovered currency speculation. We show first that the moving-average strategy popular in currency trading can be related to our ARMA(1,1) specification. We then use the first one-third of our sample to develop estimates of the returns process which we employ to calculate the optimal filter for an $\operatorname{AR}(1)$, an $\operatorname{ARMA}(1,1)$, and two representative MA returns processes. The optimal filter is obtained from the theoretical model for the uniform distribution but also numerically for the normal distribution and the bootstrapping distribution. 
Section III then conducts the out-of-sample test with the final two-thirds of our sample to compare mean returns from a switching strategy before and after transaction costs. The switching strategies are conducted under a variety of filters, including the optimal ones, for each of the $\operatorname{AR}(1), \operatorname{ARMA}(1,1)$, and MA returns cases. Section IV concludes the paper.

\section{The Theoretical Model}

\section{A. Autoregressive Conditional Returns and Two Risky Assets}

An individual investor attempts to maximize the discounted expected value of an investment over the infinite horizon. There is a fixed proportional transaction cost and the investor chooses in each period between two assets that have autocorrelated mean returns. The two assets are treated symmetrically in that shorting asset 1 is equivalent to holding asset 2 and vice versa. Each period, the investor is assumed to take a notional \$1 long position in one asset and a notional \$1 short position in the other asset. As an example, one can consider a hedge fund which allocates a given pool of funds and distributes the gains in each period back to the shareholders.

The problem can be solved by using an autonomous stochastic dynamic programming approach with state variables indicating: (1) the asset currently held (asset 1 or 2), and (2) the variables summarizing the state of the forecast variable, $x$, that characterizes the persistent part in the returns of assets 1 and 2; $x$ is assumed to follow an $\operatorname{ARMA}(1,1)$ process as a parsimonious parameterization of mild return predictability. The solution is characterized in general and is given in closed form when the shock to expected return is uniformly distributed. 
The decision problem is as follows:

$$
\begin{array}{r}
V_{i}\left(x_{t-1}, \varepsilon_{t-1}\right)=E_{t-1} r_{i}\left(x_{t}, \eta_{i t}\right)+E_{t-1} \operatorname{Max}\left[\frac{1}{1+r} V_{i}\left(x_{t}, \varepsilon_{t}\right), \frac{1}{1+r} V_{j}\left(x_{t}, \varepsilon_{t}\right)-c\right], \\
\text { for } i, j \in\{1,2\}, j \neq i
\end{array}
$$

subject to:

$$
x_{t}=\rho x_{t-1}-\delta \varepsilon_{t-1}+\varepsilon_{t}, E_{t-1} \varepsilon_{t}=0, \rho \neq \delta \text {, }
$$

where $r$ is the discount rate. The disturbance term $\varepsilon_{t}$ is assumed to have a symmetric and unimodal probability density function denoted by $f\left(\varepsilon_{t}\right)$. Each period, the investor chooses whether to invest a fixed amount (one unit) in asset 1 or asset 2 depending on which asset is held currently ( $i$ equals 1 or 2 ). A transaction cost $c$ is incurred whenever there is a switch from one asset to the other. ${ }^{3}$ The return of asset $i$ is given by $r_{i}\left(x_{t}, \eta_{i t}\right)$, with a distribution that is time-varying based on the autoregressive state variable $x_{t}$ described by equation (2); $\eta_{i t}$ is white noise. Thus, the value function at time $t-1$ depends on the state as given by the holdings of asset $i$ and the variables describing the distribution of $x_{t}$, namely $x_{t-1}$ and $\varepsilon_{t-1}$. It equals the expected return depending on current asset holdings plus the discounted expected value in the next period which depends on the return state variable $x_{t}$ as well as the choice of holdings after $x_{t}$ is revealed and minus the up-front adjustment cost that is incurred when asset holdings are switched from $i$ to $j$.

The optimal asset choice depends purely on the difference between the two assets rather than on

\footnotetext{
${ }^{3}$ Given the assumed risk neutrality, symmetry, and proportional transaction costs, intermediate positions, with investment in both assets or neither asset, are never optimal.
} 
their common levels. Hence, we can specify parsimoniously and with little loss of generality:

$$
E_{t-1}\left[r_{2}\left(x_{t}, \eta_{2 t}\right)\right]-E_{t-1}\left[r_{1}\left(x_{t}, \eta_{1 t}\right)\right]=E_{t-1} x_{t} \equiv \mu_{t}
$$

The implied general restrictions on the $r_{i}\left(x_{t}, \eta_{i t}\right)$ are that $r_{1}\left(x_{t}, \eta_{1 t}\right)=-1 / 2 x_{t}+g\left(x_{t}\right)+\eta_{1 t}$ and $r_{2}\left(x_{t}, \boldsymbol{\eta}_{2 t}\right)=1 / 2 x_{t}+g\left(x_{t}\right)+\boldsymbol{\eta}_{2 t}$ with $g(x)$ time-invariant but otherwise unrestricted. To rule out unbounded profits, it is sufficient to assume that $\mu_{t}$ is finite for all $t$.

It is now convenient to define the "difference-in-value" function:

$$
V\left(\mu_{t}\right) \equiv V_{2}\left(x_{t-1}, \varepsilon_{t-1}\right)-V_{1}\left(x_{t-1}, \varepsilon_{t-1}\right), \quad \mu_{t}=\rho x_{t-1}-\delta \varepsilon_{t-1},
$$

where the conditional expected excess return $\mu_{t}$ is defined in equation (3) and its expression is obtained from equation (2). The Appendix shows formally that the one state variable $\mu_{t}$ is sufficient to capture the dynamics related to the difference-in-value function.

The Appendix also shows that equation (4) for the difference between holding asset 2 and holding asset 1 can be written as:

$V\left(\boldsymbol{\mu}_{t}\right)=\boldsymbol{\mu}_{t}+\int_{\varepsilon_{2 t}^{*}}^{\varepsilon_{1 t}^{*}} \frac{1}{1+r} V\left(\boldsymbol{\mu}_{t+1}\right) f\left(\boldsymbol{\varepsilon}_{t}\right) d \boldsymbol{\varepsilon}_{t}+c\left[\int_{\varepsilon_{1 t}^{*}}^{\infty} f\left(\boldsymbol{\varepsilon}_{t}\right) d \boldsymbol{\varepsilon}_{t}-\int_{-\infty}^{\varepsilon_{2 t}^{*}} f\left(\boldsymbol{\varepsilon}_{t}\right) d \boldsymbol{\varepsilon}_{t}\right]$

with 


$$
\mu_{t+1}=\rho \mu_{t}+(\rho-\delta) \varepsilon_{t}
$$

as follows from equation (2).

The optimal choice of the critical values $\varepsilon_{1 t}^{*}$ and $\varepsilon_{2 t}^{*}$ is found by differentiating the right-hand side of equation (5) using Leibnitz's rule, and is given by

$$
\frac{1}{1+r} V\left[\rho \mu_{t}+(\rho-\delta) \varepsilon_{1 t}^{*}\right]=c,
$$

and

$$
\frac{1}{1+r} V\left[\rho \mu_{t}+(\rho-\delta) \varepsilon_{2 t}^{*}\right]=-c
$$

Intuitively, in equation (7), the investor is indifferent between switching assets and continuing to hold asset 1 if the discounted gain in value from switching is exactly equal to the up-front transaction cost. Equation (8) has a similar interpretation. Inverting the difference-in-value function yields for the critical values $\varepsilon_{1 t}^{*}=\left\{V^{-1}[c(1+r)]-\rho \mu_{t}\right\} /(\rho-\delta)$ and $\varepsilon_{2 t}^{*}=\left\{V^{-1}[-c(1+r)]-\rho \mu_{t}\right\} /(\rho-\delta)$.

Given equations (1) and (4) and the symmetry of the error density we have

$$
V\left(\boldsymbol{\mu}_{t}\right)=-V\left(-\boldsymbol{\mu}_{t}\right)
$$

Thus, given equation (7), (8), and (9), we have 


$$
\varepsilon_{1 t}^{*}=-\varepsilon_{2 t}^{*}-\frac{2 \rho \mu_{t}}{\rho-\delta}
$$

Defining the critical mean returns, $\mu_{1}^{*}$ and $\mu_{2}^{*}$, as $\frac{1}{1+r} V\left(\mu_{1}^{*}\right)=c$ and $\frac{1}{1+r} V\left(\mu_{2}^{*}\right)=-c$, we have $\mu^{*} \equiv \mu_{1}^{*}=-\mu_{2}^{*}=V^{-1}[c(1+r)]$. Hence, although the critical shocks $\varepsilon_{1 t}^{*}$ and $\varepsilon_{2 t}^{*}$ are time dependent, the critical mean excess returns are constant over time. Given that $V(0)=0$ and $V^{\prime}\left(\mu_{t}\right)>1$ :

$$
0<\mu^{*}<c(1+r)
$$

Summarizing and expanding the above results, the Appendix proves the following:

Proposition 1. For the decision problem in equations (1) - (3) and the definitions of $\mathrm{V}(\boldsymbol{\mu})$ and $\mu^{*}$ above and given that $f\left(\varepsilon_{t}\right)$ has unbounded support:

(a) For $\rho>0: \mathrm{V}^{\prime}(\mu)>1 \forall \mu$, and $\mathrm{V}^{\prime \prime}(\boldsymbol{\mu}) \geq(\leq) 0 \leftrightarrow(\rho-\delta) \mu \leq(\geq) 0$.

(b) $\mathrm{V}(\boldsymbol{\mu})=-\mathrm{V}(-\boldsymbol{\mu}) \forall \boldsymbol{\mu}$.

(c) $\exists$ unique $\mu^{*}: 0<\mu^{*}<c(1+r)$.

(d) $\mu^{*}=\rho \mu_{t}+(\rho-\delta) \varepsilon_{1 t}^{*}, \varepsilon_{1 t}^{*}=-\varepsilon_{2 t}^{*}-\frac{2 \rho \mu_{t}}{\rho-\delta}, \mu^{*}=V^{-1}[c(1+r)]$.

(e) The investor buys asset 2 and shorts asset 1 if $\mu_{t+1}>\mu^{*}$ and vice versa if $\mu_{t+1}<-\mu^{*}$. If $\mu^{*} \leq \mu_{t+1} \leq-\mu^{*}$, the current position is maintained. 
Proof. See Appendix.

Result (a) implies that, if profit opportunities have positive persistence, then the value function has a slope that is everywhere larger than one and is concave for positive $(\rho-\delta) \mu_{t}$ and convex for negative $(\rho-\delta) \mu_{t}$. The slope exceeds one because an increase in the expected return difference has an immediate one-to-one effect on the differential between holding asset 2 and holding asset 1 plus an additional future positive effect due to the persistence of the expected return differential that applies if there is no switching which happens with positive probability. The concavity (convexity) for $(\boldsymbol{\rho}-\boldsymbol{\delta}) \boldsymbol{\mu}_{t} \geq(\leq) 0$ arises because the higher the expected return differential is, the more incentive there is for the trader to switch from the asset with the lower mean to that with the higher mean at the fixed transaction cost, thus mitigating the expected return difference between the two positions.

Using an induction argument and the symmetry of $f\left(\varepsilon_{t}\right)$ in equation (2), result (b) follows. The reason is the symmetry of the two assets: holding asset 1 given mean return difference of $\mu_{t}$ is no different from holding asset 2 given mean return difference of $-\mu_{t}$.

Given the larger than unitary slope of the excess value function it follows that there is exactly one bounded value of $\mu^{*}$ so that equation (7) holds. Result (c) also states that $\mu^{*}$ must be positive. This is so because $V(0)=0$ from result (b) so that the positive slope of $V(\boldsymbol{\mu})$ implies that equation (7) can only hold at $\mu^{*}>0$; similarly, the larger than unitary slope of $V(\boldsymbol{\mu})$ implies that equation (7) can only hold at $\mu^{*}<c(1+r)$. Figure 1 illustrates the results in Proposition 1 for the empirically relevant case when $\rho>0$ 
and $\rho-\delta>0$.

Redefine the last two terms on the right-hand side of equation (5) for convenience, using Proposition 1d:

$$
V\left(\boldsymbol{\mu}_{t}\right)=\boldsymbol{\mu}_{t}+B\left(\boldsymbol{\mu}_{t}, \boldsymbol{\mu}^{*}\right)+C\left(\boldsymbol{\mu}_{t}, \boldsymbol{\mu}^{*}\right)
$$

By converting from the left tail of the distribution to the right tail, we can write:

$$
C\left(\boldsymbol{\mu}_{t}, \mu^{*}\right)=c\left[\int_{\varepsilon_{1 t}^{*}}^{-\varepsilon_{2 t}^{*}} f\left(\boldsymbol{\varepsilon}_{t}\right) d \boldsymbol{\varepsilon}_{t}\right]=c\left[\int_{\frac{\mu^{*}-\rho \mu_{t}}{\rho-\delta}}^{\frac{\mu^{*}+\rho \mu_{t}}{\rho-\delta}} f\left(\boldsymbol{\varepsilon}_{t}\right) d \boldsymbol{\varepsilon}_{t}\right] .
$$

The term $C\left(\mu, \mu^{*}\right)$ is interpreted as the expected transaction costs due to switching from asset 1 to asset 2 minus the expected transaction cost due to switching from asset 2 to asset 1 .

The term $B\left(\mu, \mu^{*}\right)$ indicates the average difference in next period's value from holding asset 2 compared to holding asset 1 , in the region where switching is not optimal, so that any expected return differential persists:

$$
B\left(\mu_{t}, \mu^{*}\right)=\frac{1}{1+r} \int_{\frac{-\mu^{*}-\rho \mu_{t}}{\rho-\delta}}^{\frac{\mu^{*}-\rho \mu_{t}}{\rho-\delta}} V\left[\rho \mu_{t}+(\rho-\delta) \varepsilon_{t}\right] f\left(\varepsilon_{t}\right) d \varepsilon_{t}
$$

$V(\mu)$ is evaluated from $-\mu^{*}$ to $+\mu^{*}$. Thus, given the symmetry of $V(\mu)$ in Figure 1 , the sign of $B\left(\mu, \mu^{*}\right)$ depends on the shape of the density function. For $\mu_{t}>0$ the positive part of $V(\mu)$ is weighted more than the negative part if the density is symmetric and unimodal (and vice versa for $\mu_{t}<0$ ). Thus, 
$B\left(\mu, \mu^{*}\right)$ is positive for $\mu_{t}>0$ unless the density is flat (as is the case for the uniform distribution) in which case $B\left(\mu, \mu^{*}\right)$ is zero.

Evaluating $V(\mu)$ at the critical expected return difference $\mu^{*}$, using equations (7) and (12) gives:

$$
\mu^{*}=c(1+r)-B\left(\mu^{*}, \mu^{*}\right)-C\left(\mu^{*}, \mu^{*}\right) \text {. }
$$

Intuitively, equation (15) indicates the expected excess return from holding asset 2 instead of asset 1 at which the investor is indifferent between staying with asset 1 or switching to asset $2, \mu^{*}$, to be equal to the up-front transaction cost with interest plus the expected transaction cost at state $\mu^{*}$ of switching back in the next period from asset 2 to 1 minus the expected transaction cost of not switching initially but switching anyway in the next period.

\section{B. Comparative Statics Results}

Given equation (15) or equation (7) characterizing the critical mean return differential and equation (5) stating the difference-in-value function, it is possible to derive the comparative statics results for all of the parameters in the model of equations (1) - (3). Note that to conduct the comparative statics in the last case we introduce a shift variable in equation (2) so that $x_{t}=\rho x_{t-1}-\delta \sigma_{\varepsilon} \xi_{t-1}+\sigma_{\varepsilon} \xi_{t}$, with $E_{t-1}\left(\xi_{t}\right)=0$ and $\sigma_{\xi}=1$. We also restrict $\rho>0$ to consider the case of positive persistence in profit opportunities and restrict $\rho-\delta>0$ for convenience and because this is the empirically relevant case in the application to follow. 
Proposition 2. For $\rho>0$ and $\rho-\delta>0$, the comparative statics results for the model of equations (1) - (3) are

(a) For the critical mean excess return $\mu^{*}$ :

$d \mu^{*} / d c>0, \quad d \mu^{*} / d r>0, \quad d \mu^{*} /\left.d \rho\right|_{\rho-\delta}<0$,

$d \mu^{*} /\left.d(\rho-\delta)\right|_{\rho}>0, d \mu^{*} / d \mu_{t}=0, d \mu^{*} / d \sigma_{\varepsilon}>0$.

(b) For the probability of no transaction at time t (assuming without loss of generality that the current position is long asset 1 and short asset 2$), F\left(\mu^{*}, \mu_{t} ; \gamma\right)=\int_{-\infty}^{\varepsilon_{t}^{*}} f\left(\varepsilon_{t}\right) d \varepsilon_{t}$, where $\gamma$ represents a generic parameter:

$$
\begin{aligned}
& \frac{d F\left(\boldsymbol{\mu}^{*}, \mu_{t} ; \gamma\right)}{d c}>0, \frac{d F\left(\mu^{*}, \mu_{t} ; \gamma\right)}{d r}>0,\left.\frac{d F\left(\mu^{*}, \mu_{t} ; \gamma\right)}{d \rho}\right|_{\rho-\delta} \geq 0\left(<0 \text { for } \mu_{t} \geq 0\right), \\
& \left.\frac{d F\left(\mu^{*}, \mu_{t} ; \gamma\right)}{d(\rho-\delta)}\right|_{\rho} \geq 0\left(>0 \text { for } \varepsilon_{1 t}^{*} \leq 0\right), \quad \frac{d F\left(\mu^{*}, \mu_{t} ; \gamma\right)}{d \mu_{t}}<0, \\
& \frac{d F\left(\boldsymbol{\mu}^{*}, \mu_{t} ; \gamma\right)}{d \sigma_{\varepsilon}} \geq 0\left(>0 \text { for } \varepsilon_{1 t}^{*} \leq 0\right) .
\end{aligned}
$$

Proof. Part (a) follows from equations (5) and (7). Write equation (7) as $\frac{1}{1+r} V\left(\mu^{*} ; \gamma\right)=c$, then $\frac{d \mu^{*}}{d \gamma}=\left[\frac{\partial[c(1+r)]}{\partial \gamma}-\frac{\partial V\left(\mu^{*} ; \gamma\right)}{\partial \gamma}\right] /\left[\frac{\partial V\left(\mu^{*} ; \gamma\right)}{\partial \mu^{*}}\right]$. Straightforward but tedious differentiation using Leibnitz's rule, applied to equation (5) then provides the result for each parameter $\gamma$. Part (b) follows 
similarly considering that $\frac{d F\left(\mu^{*}, \mu_{t} ; \gamma\right)}{d \gamma}=\left[\frac{\partial F\left(\mu^{*}, \mu_{t} ; \gamma\right)}{\partial \gamma}+\frac{\partial F\left(\mu^{*}, \mu_{t} ; \gamma\right)}{\partial \mu^{*}} \frac{d \mu^{*}}{d \gamma}\right]$.

The intuition for the first two results is that an increase in transaction costs or an increase in the discount/interest rate both make it more costly to adjust assets, causing the critical mean return differential to be higher to give more of an advantage to current holdings before switching occurs. Thus, the probability of no switch increases.

An increase in persistence of the mean return differential $\rho$, keeping constant the difference ( $\rho$ - ä) leads to a quicker adjustment: even if the current mean return differential foregone by not switching is currently fairly small, a larger part of it is likely to persist in future periods as follows from equation (6), implying a larger total gain from switching now. The negative effect on the probability of no transaction is reinforced when $\mu_{t} \geq 0$ because an increase in $\rho$ further implies that any current $\mu_{t}$ is more persistent, increasing the chance that a shock in the next period exceeds a given critical mean; when $\mu_{t}<0$, however, the effect of $\rho$ on the probability becomes ambiguous.

An increase in $(\rho-\ddot{a})$ for a given $\rho$ raises the critical mean return differential. The reason follows from equation (6) which implies that the conditional expected return differential adjusts more rapidly with larger $(\rho-\ddot{a})$ so that a given mean excess return is less of a signal for the future. The probability of no transaction rises unambiguously if $\varepsilon_{1 t}^{*} \leq 0$ since the direct effect of larger $(\rho-\delta)$ is to lower the absolute value of $\varepsilon_{1 t}^{*}$ raising the probability of no trade.

The level of the current conditionally expected excess return $\mu_{t}$ has no impact on the critical expected return differential. This is so because the realization of the expected return differential is sufficient 
to capture the state, no matter what value $\mu_{t}$ is, as follows by updating equation (6) by one period. The probability of no transaction, on the other hand, is affected-it decreases since less of a shock will be necessary to induce a transaction.

Lastly, an increase in the standard deviation of the innovation in the mean return differential raises the critical mean return differential and the probability of no transaction. This effect is formally similar to an increase in $(\rho-\ddot{a})$. Note that the ambiguity arises because the increase in standard deviation causes extreme changes in the mean return differential to become more likely, even though the increase in $\sigma_{\varepsilon}$ raises $\mu^{*}$, making a switch less likely.

\section{Closed Form Solution for Uniform Innovations}

It is difficult to obtain an explicit analytical solution for the optimal filter in equation (15) because, from equation (14), $B\left(\mu, \mu^{*}\right)$ depends on the value function which is of unknown functional form. However, for the special case of a constant density over the relevant range (a uniform distribution), the $B\left(\mu, \mu^{*}\right)$ term simplifies substantially, as shown in the following, so that an explicit solution can be obtained.

Assume a uniform distribution for $\varepsilon$ over the interval $[-z, z]$ with implied density $f(\varepsilon)=1 /(2 z)$. Proposition 1 requires minor modifications to apply in this case, which we omit for brevity, since the uniform distribution is bounded and not strictly unimodal. Equation (13) becomes: 


$$
C\left(\boldsymbol{\mu}_{t}, \boldsymbol{\mu}^{*}\right)=\int_{\operatorname{Max}\left(-z, \frac{\mu^{*}-\rho \mu_{t}}{\rho-\delta}\right)}^{\operatorname{Min}\left(z, \frac{\mu^{*}+\rho \mu_{t}}{\rho-\delta}\right)} f\left(\varepsilon_{t}\right) d \varepsilon_{t}=\frac{c}{2 z}\left[\operatorname{Min}\left(z, \frac{\mu^{*}+\rho \mu_{t}}{\rho-\delta}\right)-\operatorname{Max}\left(-z, \frac{\mu^{*}-\rho \mu_{t}}{\rho-\delta}\right)\right]
$$

Equation (14) becomes:

$$
B\left(\mu_{t}, \mu^{*}\right)=\frac{1}{2 z(1+r)} \int_{\operatorname{Max}\left(-z, \frac{-\mu^{*}-\rho \mu_{t}}{\rho-\delta}\right)}^{\operatorname{Min}\left(z, \frac{\mu^{*}-\rho \mu_{t}}{\rho-\delta}\right)} V\left[\rho \mu_{t}+(\rho-\delta) \varepsilon_{t}\right] d \varepsilon_{t}
$$

Note that the Min and Max operators appear because $f(\varepsilon)=0$ outside of the domain $[-z, z]$.

Proposition 3. If $\varepsilon$ is uniformly distributed over the interval $[-z, z]$ and

$z \geq \frac{[1+r(1+\rho)] c}{\rho-\delta}$

then the critical mean return differential in the model of equations (1) - (3) is given by

$$
\mu^{*}=\frac{(1+r) c}{1+\{\rho c /[(\rho-\delta) z]\}} .
$$

Proof. If $z \geq(1+\rho) \mu^{*} /(\rho-\delta)$, then the bounds in $B\left(\mu^{*}, \mu^{*}\right)$ and $C\left(\mu^{*}, \mu^{*}\right)$ are interior. Hence: 
$B\left(\mu^{*}, \mu^{*}\right)=0$ by Proposition $1 b$ and equation (17), and $C\left(\mu^{*}, \mu^{*}\right)=c \rho \mu^{*} /[(\rho-\delta) z]$ from equation

(16). Equation (15) then implies equation (19). Equations (18) and (19) in turn imply the premise that $z \geq(1+\rho) \mu^{*} /(\rho-\delta)$

Note that the assumption of the uniform distribution leads to relatively high values for $\mu^{*}$ because it causes $B\left(\mu^{*}, \mu^{*}\right)$ to be equal to its minimum value. ${ }^{4}$ Equation (19) states that given $(\rho-\delta)$ fixed, the optimal filter as a fraction of the transaction cost (plus interest) depends negatively on the persistence of the mean of the return process, $\tilde{n}$ : the trader should be willing to switch his position more readily toward a profitable opportunity if it is likely to persist longer. Considering equation (6) and the fact that for the uniform distribution $z=\sqrt{3} \sigma_{\varepsilon}$, the optimal filter depends positively on the variability of the mean of the return process, $(\boldsymbol{\rho}-\boldsymbol{\delta}) z$, scaled by the transactions cost, $c$ : if the mean is highly variable compared to the transaction cost, then a trader should require a higher immediate expected return before switching since there is a higher chance that he may want to switch back soon.

\section{Empirical Illustration for Foreign Exchange Trading: Optimal Filter Calculation}

The model developed in the preceding section can be interpreted in three different ways. First, if

\footnotetext{
${ }^{4}$ It is possible to obtain a closed form solution for the value function as well, but this depends again on the domain of the innovation distribution. In general: $V(\mu)=\mu+[\rho c /(\rho-\delta) z] \mu$ for $-\left[(\rho-\delta) z-\mu^{*}\right] / \rho \leq \mu \leq\left[(\rho-\delta) z-\mu^{*}\right] / \rho$. The condition on $\mu$ guarantees that the bounds in $B\left(\mu, \mu^{*}\right)$ and $C\left(\mu, \mu^{*}\right)$ are interior. From equation (2): $\mu_{\max }=(\rho-\delta) z /(1-\rho)$. Hence, the above equation applies to all possible states $\mu$ only if $(\rho-\delta) z \geq \mu^{*}+\rho \mu_{\max }$, or if $c \leq \frac{(1-2 \rho)(\rho-\delta) z}{(1-\rho)^{2}+\rho^{2}+r(1-\rho)}$ which requires that $\rho<\frac{1}{2}$. Note however that, even if there is no simple expression for the value function for a range of values of $\mu$, if this equation is violated, it is still appropriate to obtain the critical value from equation (19) as long as equation (18) holds.
} 
we ignore the underlying $x_{t}$ process in equation (2), the $\mu_{t}$ in equation (6) may represent an excess return that is fully known at time $t$. Thus, we are dealing with a case of pure arbitrage where the trader optimizes the after-transaction-cost excess return $V\left(\boldsymbol{\mu}_{t}\right)$. In a fully efficient market we may then expect $V\left(\boldsymbol{\mu}_{t}\right)=0 .^{5}$ Second, we may think of $\boldsymbol{\mu}_{t}$ as the risk-adjusted expected excess return, so that positive $V\left(\boldsymbol{\mu}_{t}\right)$ represents an average excess return adjusted for systematic risk. Similarly, $\mu_{t}$ could represent a particular expected utility level, which would also account for risk. Thirdly, we can interpret $\mu_{t}$ as an expected return in a case where risk is negligible or non-systematic.

Empirically, it is difficult to find accurate data to examine the first interpretation, while the second interpretation requires employing a particular risk model. Accordingly, we adopt the third interpretation of the theory in considering uncovered interest speculation in the dollar-yen spot foreign exchange market.

\section{A. A Parametric Moving Average Trading Strategy}

As discussed extensively in the literature, see for instance Frankel and Froot (1990), LeBaron (1998, 1999), Lee and Mathur (1996), Levich and Thomas (1993), Qi and Wu (2002), Sweeney (1986), and others, profitable trading strategies in foreign exchange markets traditionally have employed movingaverage (MA) technical trading rules. ${ }^{6}$ MA trading rules of size $N$ work as follows: calculate the moving average using $N$ lags of the exchange rate. Buy the currency if the current exchange rate exceeds this

\footnotetext{
${ }^{5}$ In fact, imposing this condition would give an alternative approach to estimating the transaction cost $c$ if we assume that, in an efficient market, the after-transaction-cost excess returns would be nonnegative (zero) only when using the optimal strategy.

${ }^{6}$ The source of the excess returns from MA strategies in foreign exchange markets is likely due to central bank intervention designed to smooth exchange rate fluctuations. See for instance Sweeney (2000) and Taylor (1982).
} 
average; short-sell the currency if the current exchange rate falls short of this average.

Defining $s_{t}$ as the log of the current-period spot exchange rate level (dollar price per yen) and $\Delta s_{t}$ as the percentage appreciation of the yen, the implicit exchange rate forecasting model behind the MA trading rule is

$$
\Delta s_{t+1}=\beta\left(s_{t}-\frac{\sum_{i=1}^{N} s_{t-i}}{N}\right)+\varepsilon_{t+1}, E_{t} \boldsymbol{\varepsilon}_{t+1}=0, \boldsymbol{\beta}>0 .
$$

For any positive $\beta$, equation (20) implies a positive expected exchange rate appreciation if the log of the current exchange rate exceeds the $N$-period MA. Hence, the decision rule based on equation (20) to buy (short) the currency if the expected appreciation is positive (negative) leads to a trading strategy equivalent to the MA trading strategy.

Equation (20) can straightforwardly be rewritten as an autoregressive process in the percentage change in exchange rate:

$$
\Delta s_{t+1}=\beta\left(\sum_{i=0}^{N-1} \frac{N-i}{N} \Delta s_{t-i}\right)+\varepsilon_{t+1},
$$

where the coefficients in the autoregression are given by the Bartlett weights.

Typical studies on technical analysis of foreign exchange do not utilize information on interest rates in computing the moving averages and do not estimate a parametric model for forecasting. To be fully consistent with our theory, we want to treat the excess return as the variable to be forecasted in the 
forecasting equation. To do so, we add the interest rate differential to the percentage change in exchange rate, so that Equation (21) becomes:

$$
x_{t+1}=\beta\left(\sum_{i=0}^{N-1} \frac{N-i}{N} x_{t-i}\right)+\varepsilon_{t+1},
$$

where, $x_{t} \equiv \Delta s_{t}+r_{t-1}^{J P}-r_{t-1}^{U S}, r_{t}^{J P}$ is the daily Japanese interest rate, and $r_{t}^{U S}$ is the daily U.S. interest rate. In other words, $x_{t}$ denotes the excess return from buying the Japanese yen (or the deviation from uncovered interest parity).

In the presence of transaction costs, the MA rule needs to be supplemented with a filter that indicates by how far the current spot rate must exceed (or fall short of) the MA in order to motivate a trade. The advantage of equation (22) is that it is parametric and, given an estimate for $\beta$, can provide a quantitative measure of the filter based on comparing expected return to the transaction cost.

To obtain analytically the optimal filter for the MA criterion in the context of the model it is necessary that equation (22) be translated to $\operatorname{ARMA}(1,1)$ format, for which the model provides the optimal filter (which is in closed form expression if the $\varepsilon_{t}$ are uniformly distributed). For the remainder of the paper, we assume the parameter restriction $\rho>\delta \geq 0$ for the $\operatorname{ARMA}(1,1)$ process (2). This restriction is satisfied for all our empirical specifications.

Inverting the ARMA(1,1) process of equation (2) yields an alternative autoregression: 


$$
x_{t+1}=(\rho-\delta) \sum_{i=0}^{\infty} \boldsymbol{\delta}^{i} x_{t-i}+\varepsilon_{t+1}
$$

Comparing equations (22) and (23) it follows that equation (23) is a good approximation for equation (22)

if we set $\rho-\delta=\beta$ and if the $\frac{N-i}{N}$ terms are close to the $\delta^{i}$ for all $i$. Taking a log approximation, and choosing $\delta$ to match the Bartlett weights:

$$
-i / N \approx \log \left(\frac{N-i}{N}\right)=i \log \delta \quad \Rightarrow \quad \delta=\exp \left(\frac{-1}{N}\right)
$$

Thus, from $\rho-\delta=\beta$ and $\delta=\exp \left(\frac{-1}{N}\right)$, we can uniquely identify a $\rho$ and $\delta$ that provide a good ARMA(1,1) proxy for any $N$-period MA-based process. In turn, the ARMA parameters allow us to calculate analytically the critical expected return $\mu^{*}$ governing the transaction choice.

\section{B. Preliminary Dollar-Yen Process Estimates}

Our data on the Japanese yen - U.S. dollar spot exchange rate covers the period from August 31, 1978 to May 3, 2003 with 6195 daily observations. Daily exchange rate data for the Japanese yen is downloaded from the Federal Reserve's webpage. For interest rates, we obtain Financial Times' Eurocurrency interest rates from Datastream International. The first 1/3 of the sample (2065 observations) is used for model estimation. Out-of-sample forecasting starts on November 28, 1986 until the end of the 
sample (4130 observations). We estimate the exchange rate dynamics in four ways: 1. an AR(1) process ( $\delta$ in equation 2 is set equal to zero); 2 . an $\operatorname{ARMA}(1,1)$ process (equation 2); 3 . a process consistent with an MA rule of 21 lags (21 trading days in a month), as is commonly considered with daily data (equation 22); 4. a process consistent with an MA rule of size 126 (half a year), which is around the size typically used by traders, although results appear to depend little on the exact size of the MA process chosen (LeBaron, 1998, 1999).

Columns (1)-(5) of Table I show the results of the in-sample model estimation using the first 1/3 of our sample for each way of capturing the exchange rate dynamics. For the AR(1) process we find low persistence with $\rho=0.0548$ and a standard error of $\sigma_{\varepsilon}=0.00659$; for the ARMA $(1,1)$ process we find $\rho=0.918$ and $\delta=0.880$ with a standard error of $\sigma_{\varepsilon}=0.00658$. Thus, both processes provide similar accuracy although the parameters differ substantially. While the data cannot tell us clearly whether the $\mathrm{AR}(1)$ or the ARMA $(1,1)$ process is better at describing the exchange rate dynamics, we will see that the implications for optimal trading are substantially different. ${ }^{7}$ For the representative moving average rule with 21 lags we find for the slope in equation (22) that $\beta=0.0257$ and $\sigma_{\varepsilon}=0.00656$. Since we have $N=21$ we obtain from equation (24) that $\rho=0.979$ and $\delta=0.954$, which is not statistically distinguishable from the direct estimates from the $\operatorname{ARMA}(1,1)$ model. The MA(126) process yields $\beta=0.00818$ and $\sigma_{\varepsilon}=$ 0.00635 , implying by approximation that $\rho=0.999$ and $\delta=0.992$.

We assume a round trip transaction cost of $c=0.001$ (10 basis points) throughout. Sweeney (1986) finds a transaction cost of 12.5 basis points for major foreign exchange markets, but more recent

\footnotetext{
${ }^{7}$ Balvers and Mitchell (1997) raise this issue in the context of optimal portfolio choice under return predictability.
} 
work by Bessembinder (1994), Melvin and Tan (1996), and Cheung and Wong (2000) finds bid-ask spreads for major exchange rates between 5 and 9 basis points. To account for transaction costs in addition to those imbedded in the bid-ask spread, related to broker fees and commissions, and the lendingborrowing interest differential we use 10 basis points as a realistic number for the dollar-yen market. The daily U.S. interest rate is on average over the first $1 / 3$ of the sample equal to 0.000439 percent. This average interest rate is used as a proxy for the discount rate $r$ in computing the optimal filter in Equation (19).

We estimate the optimal filter $\mu^{*}$ using three methods. Firstly, under the assumption that the error term $\varepsilon_{t}$ is uniformly distributed the optimal filter, denoted by $\mu_{v}^{*}$, can be analytically calculated using equation (19). Secondly, $\varepsilon_{t}$ is assumed to follow a normal distribution. In this case, the result in equation (19) no longer holds, and we estimate the optimal filter, denoted by $\mu_{N}^{*}$, through Monte-Carlo simulation. Lastly, we do not make an assumption about the distribution of $\varepsilon_{t}$ and estimate the optimal filter, denoted by $\mu_{B}^{*}$, by bootstrapping the model residuals $\hat{\varepsilon}_{t}$ with replacement.

\section{The Optimal Filter Implied by the Theory under the Uniform Distribution}

Under the assumption of a uniform distribution, we can obtain $z$ from the relation $z=\sqrt{3} \sigma_{\varepsilon}$. All the information now is there to allow us to calculate the optimal filter from equation (19) for the dollar-yen exchange rate. Column (6) of Table I provides the results. For the AR(1) case we find that the ratio of the critical return to the transaction cost is $\mu_{U}^{*} / c=0.92$. Hence, in this case the optimal filter is not very different from a naï ve filter that equals the transaction $\operatorname{cost} c$. The main reason is that, from equation (6), 
the persistence in the mean return is small at $\rho=0.0548$ so that, no matter what the current holdings are, there is not much difference in future probabilities of trading.

On the other hand, for the $\operatorname{ARMA}(1,1)$ case with $\rho=0.918$ and $\delta=0.880$, we find that the optimal filter is dramatically different at $\mu_{U}^{*} / c=0.32$. The reason that this number is so much lower than under the $\operatorname{AR}(1)$ case is clear from equation (6). The persistence is not only high now with $\rho=0.918$ but it is also high relative to the innovation in the conditional mean that is $(\rho-\delta) \sigma_{\varepsilon}=0.038 \sigma_{\varepsilon}$. Hence, it is highly likely that the exchange position (dollar or yen) with the currently positive expected return is going to be unchanged in the nearby future.

For the 1-month MA process, the parameters backed out from the MA(21) model yield $\mu_{U}^{*} / c$ $=0.23$. Note that inequality (18) is violated, as is necessary when $\mu_{U}^{*} / c<0.50$, implying that the analytical value obtained from equation (19) is no longer accurate and must be viewed as a good approximation, hence it is more precise to state that $\mu_{U}^{*} / c \approx 0.23$. Intuitively, the slow adjustment in the conditional mean for these parameter values implies that, in some cases, even the most extreme realization of the exchange rate innovation would not be sufficient to induce switching. Hence, one would be certain of avoiding transaction costs for at least one period (and likely more) by buying or keeping the exchange with the positive expected return. This explains of course the low value of the critical expected return relative to the transaction cost.

The 6-month MA process, $\mathrm{MA}(126)$ yields the smallest filter, $\mu_{U}^{*} / c=0.083$. One reason is the high persistence of expected return (the implied persistence parameter $\rho=0.999$ ). Another is the fact that by nature the long MA process is very smooth so changes in the mean occur very slowly so that the number 
of transactions is small, even when there is no filter. This is undoubtedly a reason for the popularity of this particular trading rule with practitioners.

\section{The Optimal Filter Obtained Numerically under the Normal Distribution}

As a check on the dependence of the results on the uniform distribution, we also find the optimal filter numerically using a Monte Carlo approach assuming normality and a bootstrapping approach.

\section{Expected trading rule returns under Normality}

Under normality it is possible, and may help build some perspective and intuition, to consider some ballpark figures of what mean returns should be expected, before and after transaction costs, based on the predictability implied by the estimates of the returns process. Given normality, equation (6) tells us that

$$
\mu_{t+1}=\rho \mu_{t}+(\rho-\delta) \varepsilon_{t}, \quad \varepsilon_{t} \sim N\left(0, \sigma_{\varepsilon}\right)
$$

Then the expected return given a trading strategy of switching between two currencies ignoring transaction costs would equal the unconditional mean given truncation at zero, since the decision rule always implies a switch to the one currency with a positive expected excess return. Following for instance Maddala (1983,

p.365) this truncated mean is given as $E\left(\mu_{t} \mid \mu_{t} \geq 0\right)=\frac{\sigma_{\mu} f(0)}{1-F(0)}$ where $f$ and $F$ indicate respectively the probability density function and cumulative distribution function of the standard normal, and $\sigma_{\mu}$ represents 
the unconditional standard deviation of $\mu_{t}$ which equals $\frac{(\rho-\delta) \sigma_{\varepsilon}}{\sqrt{1-\rho^{2}}}$. Thus, multiplying by 252 trading days to annualize:

$$
E\left(\mu_{t} \mid \mu^{*}=0\right)=2 \cdot 252 \cdot \sigma_{\mu} \cdot f(0), \quad \sigma_{\mu}=\frac{(\rho-\delta) \sigma_{\varepsilon}}{\sqrt{1-\rho^{2}}}
$$

Taking our parameter estimates and given that $f(0)=0.399$ we obtain for the $\mathrm{AR}(1)$ process an expected return of $E\left(\mu_{t} \mid \mu^{*}=0\right)=7.28$ percent and for the ARMA(1,1) process $E\left(\mu_{t} \mid \mu^{*}=0\right)=12.80$ percent. Given a roundtrip transaction cost of 10 basis points this implies a break-even point of 73 transactions a year if the $\mathrm{AR}(1)$ process holds and of 128 transactions a year if the ARMA(1,1) process holds. If we ignore persistence (the profit opportunities are white noise) and consider the trading strategy without filter then the probability of a switch is 50 percent, implying an average number of roundtrip transactions of 126 a year so that expected returns after transaction costs become negative for the AR(1) process and zero for the $\operatorname{ARMA}(1,1)$ process.

\section{Monte Carlo Simulation}

While these numbers are suggestive, the approximations used here unfortunately do not properly consider the persistence in mean returns and would be a poor guide for an optimal filter. Deriving and accounting for the distribution of actual returns according to equation (25) conditional on a filter is extremely difficult. This is of course the reason that we resort to the uniform distribution for analytical solutions and why we use simulation to obtain the optimal filters for the normal distribution numerically.

For each Monte-Carlo trial, we simulate expected returns $\mu_{t}$ using Equation (25) with parameters 
estimated from the first $1 / 3$ of the sample. We then choose the filter $\mu_{N}^{*}$ which maximizes the after-cost average excess return. This process is replicated 500 times. Column (7) of Table I reports the median value of the optimal filter to transaction cost ratio, $\mu_{N}^{*} / c$, over the 500 Monte-Carlo trials. For each model, the ratio $\mu_{N}^{*} / c$ is quite close to the optimal ratio implied under the uniform distribution $\mu_{U}^{*} / c$, with the difference between them never exceeding $5 \%$ of the transaction cost.

\section{E. The Optimal Filter Obtained Numerically under Bootstrapping}

The actual distribution of $\varepsilon_{t}$ may be neither uniform nor normal. In this case, we re-sample with replacement the fitted residuals $\hat{\varepsilon}_{t}$ of Equation (25) and use model parameters to generate expected return observations $\mu_{t}$. Similar to the Monte-Carlo experiment, for each bootstrapping trial, the optimal filter is chosen to be the one which maximizes the after-cost average excess return. Column (8) of Table I reports the median estimate of the optimal filter to cost ratio $\mu_{B}^{*} / c$ over 500 bootstrapping replications. Encouragingly, the optimal filters, for the theoretical uniform distribution case and the numerical normal and bootstrapping cases, are quite similar for each of the returns processes. Thus, the optimal filter value is robust to distributional assumptions.

Applying the optimal filters to the data in trading on deviations from uncovered interest parity, we expect straightforwardly that the optimal filters will outperform naï ve filters. In particular, we expect that the optimal filter does better than the naï ve " 0 " filter that is used implicitly when transaction costs are ignored for trading decisions (but not for calculating returns), because it saves on transaction costs; and does better than the naï ve "c" filter that is employed when trading costs are considered myopically, 
because it does not filter out as many profitable transactions.

\section{Out-of-Sample Optimal Switching Strategy Results}

We start our first-day forecast on November 28, 1986 (after the first one third of the sample). For each of the four exchange rate return specifications, we estimate the model parameters using all observations for the first one third of the sample (up to November 27, 1986) and make the first forecast (for November 28). If the forecasted excess return (the excess return is defined as the difference between the return from holding the Japanese yen, which is the percentage exchange rate change plus the one-day Japanese interest rate, and the return from holding the U.S. dollar, which is the one-day U.S. interest rate) is positive, we take a long position in the Japanese yen, and simultaneously take a short position in the dollar. Conversely, if the forecasted excess return is negative, we take a long position in the dollar and a short position in the yen. The difference in returns between the long and short positions (Long-Short) represents the excess return from a zero-cost investment strategy.

From the second forecasted day (November 29) until the end of the sample, our strategy works as follows. For each day, we use all available observations to estimate the model parameters and forecast the excess return for the following day. If either of the following two conditions occurs, a transaction will take place. (1) If the forecasted excess return is positive, its magnitude is larger than the transaction cost filter, and we currently have a long position in the dollar (and a short position in the yen), then we reverse our position by taking a long position in the yen and a short position in the dollar for the following day. This 
counts as one trade involving two round-trip transaction costs. ${ }^{8}$ (2) If the forecasted excess return is negative, its magnitude is larger than the transaction cost filter, and the current holdings are long in the yen and short in the dollar, then we reverse our position by taking a long position in the dollar and a short position in the yen. This counts as one transaction and again involves two round-trip costs. If neither of the above two conditions applies, no trade will take place. The current holdings (both long and short) carry over to the following day and no transaction costs are incurred.

We compute the average return and the associated $t$-ratio for each strategy for the out-of-sample forecasting period. We document the before-cost and after-cost return rates for the case without a filter; and the after-cost return rates for the cases with transaction cost filters. Panel A of Table II reports the results for the $\mathrm{AR}(1)$ forecasting model. Without imposing a transaction cost filter, the strategy involves 2,100 switches over 4,130 trading days (over 50 percent of the time). In the absence of transaction costs, the strategy produces an annualized excess return of 6.9 percent with a $t$-ratio of 2.486 which is statistically significant at the 5 percent level using a 2-sided test. (This number is quite close to the 7.3 percent return expected for an $\mathrm{AR}(1)$ process under the normality assumption). However, a round-trip cost of 10 basis points completely wipes out the profits, resulting in a negative excess return of 18.8 percent. A naï ve filter equal to the actual transaction cost of 10 basis points dramatically reduces the number of transactions to 34, and yields an insignificant excess return of 0.9 percent per annum. While it is somewhat useful, this naï ve filter may be too conservative because it does not exploit the information on the persistence of

\footnotetext{
${ }^{8}$ The transaction $\operatorname{cost} c$ in the theory is equivalent to the cost of one round-trip as follows from equation (1) where $c$ represents the cost of switching from a long position in currency $i$ to a long position in currency $j$. The empirical case, however, is based on the difference-in-value function in equation (5) in which a long and a short position are combined, implying indeed two roundtrips when a transaction occurs.
} 
expected return in the exchange rate, thereby missing a number of profitable trades. The strategy with the optimal filter $\mu_{U}^{*}$ under the assumption of a uniform distribution captures just that opportunity. It produces 42 trades and yields a higher excess return of 5.6 percent which is significant at the 5 percent level. Similarly, the optimal filter under the normality assumption $\mu_{N}^{*}$ produces an average excess return of 5.3 percent per annum which is significant at the 10 percent level. The bootstrapped filter $\mu_{B}^{*}$ yields an insignificant excess return of 2.2 percent.

Panel B reports the results for the ARMA(1,1) model. The after-cost excess return for the case without a filter is 0.3 percent with 444 trades. The naï ve filter equal to " $c$ " dramatically reduces the number of trades to 12 , resulting in an insignificant excess return of 0.5 percent. In contrast, the optimal filter, $\mu_{U}^{*}$, captures many of the profitable trades and yields an excess return of 5.1 percent which is significant at the 10 percent level. The filter under the bootstrapped distribution $\mu_{B}^{*}$ produces nearly the same results as $\mu_{U}^{*}$, whereas the filter under the normality assumption, $\mu_{N}^{*}$, yields a higher excess return of 6.6 percent which is significant at the 5 percent level.

For the MA(21) model reported in Panel C, our strategy with the optimal filters again generates higher excess returns than the alternatives, although none of the excess returns are statistically significant.

Finally, the long MA(126) process provides very smooth forecast of expected returns. While the strategy without filter yields an after-cost return of 4.9 percent which is significant at the 10 percent level, the naï ve filter equal to $c$ skips too many profitable trades, resulting in a negative return of 2 percent. The optimal filter $\mu_{U}^{*}$, while very small relative to $c$, is capable of filtering many days with low expected returns and capturing those days when expected returns are substantial. This filter produces an expected return of 
5.7 percent which is significant at the 5 percent level. The other two filters, $\mu_{N}^{*}$ and $\mu_{B}^{*}$ yield somewhat smaller returns.

Figures 2 through 5 display the trading strategy returns (after cost) and trading costs for the four return processes as a function of the filter value. As expected, the trading cost declines monotonically as the filter value rises. The after-cost excess return lines illustrate that in all cases the ex ante optimal filters are reasonably close to the ex post optimum. Since the actual data is just one random draw from the unobserved true process this is all one should expect of a good model. Except for the AR(1) case, the trading strategy returns display the hump-shaped pattern expected for the after-cost returns.

A striking feature of these four figures is that, even though the optimal filters differ dramatically across the four cases, the empirical maximum filter value is quite close to the optimal filter in all four cases. While each case approximates the true data process to a certain extent, it is not surprising that the $\operatorname{ARMA}(1,1)$ process provides the best overall fit as it is well-known to be a parsimonious description of general $\operatorname{ARMA}(\mathrm{p}, \mathrm{q})$ processes.

\section{Conclusion}

If transitory profitable trading opportunities exist, filter rules are used in practice to mitigate transaction costs. The filter size is difficult to determine a priori. This paper uses a dynamic programming framework to design a filter that is optimal in the sense of maximizing expected returns after transaction costs. The optimal filter size depends negatively on the degree of persistence of the profitable trading opportunities, positively on transaction costs, and positively on the standard deviation of shocks.

We apply our theoretical results to foreign exchange trading by parameterizing the moving average 
strategy often employed in foreign exchange markets. The parameterization implies the same decisions as the moving average rule in the absence of transaction costs, but has the advantage of translating the buy/sell signal into the same units as the transaction costs so that the optimal filter can be calculated.

Application to daily dollar-yen trading demonstrates that the optimal filter can differ dramatically from a naï ve filter equal to the transaction cost. This depends importantly on the time series process that we assume for the exchange rate dynamics. In particular, we find that for an $\operatorname{AR}(1)$ process the optimal filter is close to the naï ve transaction cost filter, but for an $\operatorname{ARMA}(1,1)$ process the optimal filter is only around 30 percent of the naï ve transaction cost filter, and for the more stable MA processes, the optimal filter is smaller still as a fraction of the transaction cost. Impressively, the optimal filters under the assumptions of uniform, normal, and bootstrap distributions are all very close to one another and all are quite close to the ex post after-cost return maximizing level.

We confirm that simple daily moving average foreign exchange trading generates positive returns that disappear after accounting for transaction costs. However, when the optimal filter is used, returns after transaction costs remain positive and are higher than for naï ve filters. This result strongly suggests that caution should be applied in dismissing abnormal returns as due to transactions costs, merely because the after-cost return is negative or insignificant. For instance, Lesmond, Schill, and Zhou (2003) argue convincingly that momentum profits disappear when actual transaction costs are properly considered, even after accounting for the proportion of securities held over in each period. But their after-cost returns are akin to those for our suboptimal zero filter strategy. It would be interesting to see what outcome would arise if an optimal filter were used.

Apart from the practical advantages of using the optimal filter, there is also a methodological 
advantage: in studies attempting to calculate abnormal returns from particular trading strategies in which transaction costs are important, there is no guideline as to what filter to use in dealing with transaction costs. Lesmond, Schill, and Zhou (2003, p.24) note: "Although we observe that trading costs are of similar magnitude to the relative strength returns for the specific strategies we consider, there is an infinite number of momentum-oriented strategies to evaluate, so we can not reject the existence of trading profits for all strategies." Rather than allowing the data mining problem that is likely to arise when a variety of filter sizes are applied, our approach here provides a first step in deriving a unique filter that can be related to observable variables. 


\section{Appendix}

\section{A. The Difference-In-Value Function}

From equations (1) and (2):

$$
\begin{array}{r}
V_{i}\left(x_{t-1}, \boldsymbol{\varepsilon}_{t-1}\right)=E_{t-1} r_{i}\left(x_{t}, \boldsymbol{\eta}_{i t}\right)+\int_{\left\{\varepsilon_{t}: \frac{1}{1+r} V_{i t}>\frac{1}{1+r} V_{j t}-c\right\}} \frac{1}{1+r} V_{i}\left(x_{t}, \boldsymbol{\varepsilon}_{t}\right) f\left(\boldsymbol{\varepsilon}_{t}\right) d \boldsymbol{\varepsilon}_{t}+ \\
\int_{\left\{\varepsilon_{t}: \frac{1}{1+r} V_{i t} \leq \frac{1}{1+r} V_{j t}-c\right\}}\left[\frac{1}{1+r} V_{j}\left(x_{t}, \boldsymbol{\varepsilon}_{t}\right)-c\right] f\left(\boldsymbol{\varepsilon}_{t}\right) d \boldsymbol{\varepsilon}_{t},
\end{array}
$$

with $x_{t}=\rho x_{t-1}-\delta \varepsilon_{t-1}+\varepsilon_{t}, \quad E_{t-1} \varepsilon_{t}=0, \quad \rho \neq \delta, f\left(\varepsilon_{t}\right)=f\left(-\varepsilon_{t}\right)$.

Taking the difference $V_{\text {diff }}(x, \varepsilon)=V_{2}(x, \varepsilon)-V_{1}(x, \varepsilon)$ and using equation (3) yields:

$$
\begin{gathered}
V_{\text {diff }}\left(x_{t-1}, \boldsymbol{\varepsilon}_{t-1}\right)=E_{t-1} x_{t}+\int_{\left\{\varepsilon_{t}: c \geq \frac{1}{1+r} V_{d i f f} \geq-c\right\}} \frac{1}{1+r} V_{\text {diff }}\left(x_{t}, \boldsymbol{\varepsilon}_{t}\right) f\left(\boldsymbol{\varepsilon}_{t}\right) d \boldsymbol{\varepsilon}_{t}+ \\
c\left[\int_{\left\{\varepsilon_{t}: \frac{1}{1+r} V_{\text {diff }}>-c\right\}} f\left(\boldsymbol{\varepsilon}_{t}\right) d \boldsymbol{\varepsilon}_{t}-\int_{\left\{\varepsilon_{t}: c>\frac{1}{1+r} V_{\text {diff }}\right\}} f\left(\boldsymbol{\varepsilon}_{t}\right) d \boldsymbol{\varepsilon}_{t}\right] .
\end{gathered}
$$

Redefining the state variables so that $V_{\text {diff }}\left(x_{t}, \boldsymbol{\varepsilon}_{t}\right) \equiv V_{d}\left(\mu_{t+1}, \boldsymbol{\varepsilon}_{t}\right)$ is without loss of generality since $\mu_{t+1} \equiv \rho x_{t}-\delta \varepsilon_{t}$ and yields:

$$
\begin{aligned}
V_{d}\left(\mu_{t}, \varepsilon_{t-1}\right)=\mu_{t}+ & \int_{\left\{\varepsilon_{t}: c \geq \frac{1}{1+r} V_{d} \geq-c\right\}} \frac{1}{1+r} V_{d}\left(\mu_{t+1}, \varepsilon_{t}\right) f\left(\varepsilon_{t}\right) d \varepsilon_{t}+ \\
& c\left[\int_{\left\{\varepsilon_{t}: \frac{1}{1+r} V_{d}>-c\right\}} f\left(\varepsilon_{t}\right) d \varepsilon_{t}-\int_{\left\{\varepsilon_{t}: c>\frac{1}{1+r} V_{d}\right\}} f\left(\varepsilon_{t}\right) d \varepsilon_{t}\right],
\end{aligned}
$$

with $\mu_{t+1}=\rho \mu_{t}+(\rho-\delta) \varepsilon_{t}, E_{t-1} \varepsilon_{t}=0, \quad \rho \neq \delta, f\left(\varepsilon_{t}\right)=f\left(-\varepsilon_{t}\right)$.

It is clear from (A3) that, once the state variable $\mu_{t}$ is considered, there is no additional role for the state 
variable $\varepsilon_{t-1}$. Thus we have

$$
V\left(\mu_{t}\right) \equiv V_{d}\left(\mu_{t}, \varepsilon_{t-1}\right)
$$

\section{B. Proof of Proposition 1}

It is easy to see that $V\left(\mu_{t}\right)$ is monotonically increasing in $\mu_{t}$ and that, in effect, for $\rho>0$ a one unit increase in $\mu_{t}$ raises $V\left(\mu_{t}\right)$ more than one unit: there is a direct one-to-one benefit of higher $\mu_{t}$ given that asset 2 is held, plus the positive persistence ( $\rho>0$ in A3) means that an additional benefit also is expected to extend to the future. If we assume that the innovation $\varepsilon_{t}{ }^{\prime} s$ has unbounded support, then since $\mu_{t+1}=\rho \mu_{t}+(\rho-\delta) \varepsilon_{t}$ and $\rho \neq \delta$, any value of $\mu_{t+1}$ is possible. Hence, a critical $\varepsilon_{t}=\varepsilon_{1 t}^{*}$ must exist such that $\frac{1}{1+r} V\left[\rho \mu_{t}+(\rho-\delta) \varepsilon_{1 t}^{*}\right]=c$ and a critical $\varepsilon_{t}=\varepsilon_{2 t}^{*}$ must exist such that $\frac{1}{1+r} V\left[\rho \mu_{t}+(\rho-\delta) \varepsilon_{2 t}^{*}\right]=-c$. Moreover, these critical $\varepsilon_{t}$ are unique due to the monotonicity of $V\left(\mu_{t}\right)$. Thus we can write:

$$
V\left(\boldsymbol{\mu}_{t}\right)=\boldsymbol{\mu}_{t}+\int_{\varepsilon_{2 t}^{*}}^{\varepsilon_{t t}^{*}} \frac{1}{1+r} V\left(\mu_{t+1}\right) f\left(\boldsymbol{\varepsilon}_{t}\right) d \boldsymbol{\varepsilon}_{t}-c\left[\int_{-\infty}^{\varepsilon_{2 t}^{*}} f\left(\boldsymbol{\varepsilon}_{t}\right) d \boldsymbol{\varepsilon}_{t}-\int_{\varepsilon_{1 t}^{*}}^{\infty} f\left(\boldsymbol{\varepsilon}_{t}\right) d \boldsymbol{\varepsilon}_{t}\right]
$$

with $\mu_{t+1}=\rho \mu_{t}+(\rho-\delta) \varepsilon_{t}, \varepsilon_{1 t}^{*}=\left\{V^{-1}[c(1+r)]-\rho \mu_{t}\right\} /(\rho-\delta)$, and

$\varepsilon_{2 t}^{*}=\left\{V^{-1}[-c(1+r)]-\rho \mu_{t}\right\} /(\rho-\delta)$.

Given (A4) and (A5) and since $f\left(\varepsilon_{t}\right)=f\left(-\varepsilon_{t}\right)$ it follows that

$$
V\left(\mu_{t}\right)=-V\left(-\mu_{t}\right)
$$

Thus, given (A6) and equations (7) and (8) we have 


$$
\varepsilon_{1 t}^{*}=-\varepsilon_{2 t}^{*}-\frac{2 \rho \mu_{t}}{\rho-\delta}
$$

Defining the critical mean returns as $\frac{1}{1+r} V\left(\mu_{1}^{*}\right)=c$ and $\frac{1}{1+r} V\left(\mu_{2}^{*}\right)=-c$, we have

$$
\mu^{*} \equiv \mu_{1}^{*}=-\mu_{2}^{*}=V^{-1}[c(1+r)] \text {. Hence, given that } V(0)=0 \text { and } V^{\prime}\left(\mu_{t}\right)>1 \text { : }
$$

$$
0<\mu^{*}<c(1+r)
$$

Concavity of $V\left(\mu_{t}\right)$ for $\mu_{t}>0$ follows from differentiation of (A5), using Leibnitz's rule:

$$
V^{\prime}\left(\mu_{t}\right)=1+\frac{\rho}{1+r} \int_{\varepsilon_{2 t}^{*}}^{\varepsilon_{t}^{*}} V^{\prime}\left(\mu_{t+1}\right) f\left(\varepsilon_{t}\right) d \varepsilon_{t}
$$

Again differentiating yields:

$$
V^{\prime \prime}\left(\mu_{t}\right)=\frac{-\rho^{2}}{(\rho-\delta)(1+r)} V^{\prime}\left(\mu^{*}\right)\left[f\left(\varepsilon_{1}^{*}\right)-f\left(\varepsilon_{2}^{*}\right)\right]+\frac{\rho^{2}}{1+r} \int_{\varepsilon_{2 t}^{*}}^{\varepsilon_{1 t}^{*}} V^{\prime \prime}\left(\mu_{t+1}\right) f\left(\varepsilon_{t}\right) d \varepsilon_{t},
$$

where we used the fact that from (A6) $V^{\prime}\left(\mu_{t}\right)=V^{\prime}\left(-\mu_{t}\right)$. Given that $f\left(\varepsilon_{t}\right)$ is symmetric and unimodal we have for $\boldsymbol{\rho}>0$ that $f\left(\varepsilon_{1}^{*}\right)>f\left(\varepsilon_{2}^{*}\right)$ if $\mu_{t}>0$ so that $V^{\prime \prime}\left(\mu_{t}\right)<0$ if $\boldsymbol{\rho}-\boldsymbol{\delta}>0$ and $V^{\prime \prime}\left(\boldsymbol{\mu}_{t}\right)>0$ if $\boldsymbol{\rho}-\boldsymbol{\delta}<0$. Thus, from (A6), for $\boldsymbol{\mu}_{t}<0$ we have $V^{\prime \prime}\left(\mu_{t}\right)>0$ if $\boldsymbol{\rho}-\boldsymbol{\delta}>0$ and $V^{\prime \prime}\left(\boldsymbol{\mu}_{t}\right)<0$ if $\boldsymbol{\rho}-\boldsymbol{\delta}<0$. 


\section{References}

Balduzzi, P., and A. Lynch, 1999, Transaction costs and predictability: some utility cost calculations, Journal of Financial Economics 52, 47-78.

Balvers, R.J., and D.W. Mitchell, 1997, Autocorrelated returns and optimal intertemporal portfolio choice, Management Science 43, 1537-1551.

Balvers, R.J., Y. Wu, and E. Gilliland, 2000, Mean reversion across national stock markets and parametric contrarian investment strategies, Journal of Finance 55, 745-772.

Bessembinder, Hendrik, 1994, Bid-ask spreads in the interbank foreign exchange markets, Journal of Financial Economics 35, 317-348.

Cheung, Y.W., and C. Y. P Wong, 2000, A survey of market practitioners' view on exchange rate dynamics, Journal of International Economics 51, 401-419.

Cooper, Michael, 1999, Filter rules based on price and volume in individual security overreaction, Review of Financial Studies 12, 901-935.

Fama, E. F., and M. Blume, 1966, Filter rules and stock market trading profits, Journal of Business 39, 226-241.

Frankel, J.A., and K.A. Froot, 1990, Chartists, fundamentalists, and trading in the foreign exchange market, American Economic Review 80, 181-185.

Grundy, Bruce, and J. Spencer Martin, 2001, Understanding the nature and the risks and the sources of the rewards to momentum investing, Review of Financial Studies 14, 29-78.

Hanna, J. D., and M. Ready, 2001, Profitable predictability in the cross-section of stock returns, mimeo, University of Chicago.

Knez, Peter J., and Mark J. Ready, 1996, Estimating the profits from trading strategies, Review of Financial Studies 9, 1121-1163.

LeBaron, B., 1998, Technical trading rules and regime shifts in foreign exchange, In Advanced Trading Rules, eds. F. Acar and S. Satchell, 5-40, Butterworth-Heinemann, Woburn, MA.

LeBaron, B., 1999, Technical trading rule profitability and foreign exchange intervention, Journal of International Economics 49, 125-143.

Lee, C. I., and L. Mathur, 1996, Trading rule profits in European currency spot crossrates, Journal of Banking and Finance 20, 949-962. 
Lesmond, David A., Michael J. Schill, and Chunsheng Zhou, 2003, The illusory nature of momentum profits, working paper, University of Virginia, forthcoming Journal of Financial Economics.

Levich, R.M., and L.R. Thomas, 1993, The significance of technical trading-rule profits in the foreign exchange market: a bootstrap approach, Journal of International Money and Finance 12, 451474.

Liu, Hong, and Mark Loewenstein, 2002, Optimal portfolio selection with transaction costs and finite horizons, Review of Financial Studies 15, 805-835.

Lynch A., and P. Balduzzi, 2000, Predictability and transaction costs: the impact on rebalancing rules and behavior, Journal of Finance 55, 2285-2310.

Maddala, G.S., 1983, Limited Dependent and Qualitative Variables in Econometrics, Cambridge University Press, New York.

Melvin, M., and K. Tan, 1996, Foreign exchange market bid-ask spreads and the market price of social unrest, Oxford Economic Papers 48, 329-341.

Qi, M., and Y. Wu, 2002, Technical trading-rule profitability, data snooping, and reality check: evidence from the foreign exchange market, mimeo, Rutgers University.

Sweeney, R.J., 1986, Beating the foreign exchange market, Journal of Finance 41, 163-182.

Sweeney, R.J., 2000, Does the Fed beat the foreign-exchange market? Journal of Banking and Finance 24, 665-694.

Taylor, D., 1982, Official intervention in the foreign exchange market, or, bet against the central bank, Journal of Political Economy 90, 356-368. 


\section{Table I Model Parameters and Implied Optimal Transaction Cost Filters}

This table reports parameter estimates for candidate forecasting models and for each model the implied optimal filter/transaction cost ratios. The forecasting models are $\operatorname{AR}(1): x_{t+1}=\rho x_{t}+\varepsilon_{t+1} ; \operatorname{ARMA}(1,1): x_{t+1}=\rho x_{t}+\varepsilon_{t+1}-\delta \varepsilon_{t}$; and the transformed model of $\mathrm{MA}(\mathrm{N}): x_{t+1}=\beta \sum_{i=0}^{N-1}\left[\left(\frac{N-i}{N}\right) x_{t-i}\right]+\varepsilon_{t+1}$, with $N=21$ and 126, where $x_{t} \equiv \Delta s_{t}+r_{t-1}^{J P}-r_{t-1}^{U S}$, $S_{t}$ is the $\log$ of the U.S. dollar price of one Japanese yen, $r_{t}^{J P}$ is the daily Japanese interest rate, and $r_{t}^{U S}$ is the daily U.S. interest rate. The full sample data covers the period from August 31, 1978 to May 3, 2003 with 6,195 daily observations. The parameters are estimated with the first $1 / 3$ of the sample (2,065 observations). The implied optimal transaction cost filters are calculated under three differential distributional assumptions of $\boldsymbol{\varepsilon}_{t}$ : uniform where the optimal filter denoted by $\mu_{U}^{*}$ is calculated using equation (19); normal, where the optimal filter denoted by $\mu_{N}^{*}$ is estimated through Monte-Carlo simulation with 500 replications; and bootstrap, where the optimal filter denoted by $\mu_{B}^{*}$ is estimated through bootstrapping with replacement with 500 replications. The round-trip transaction $\operatorname{cost} c=0.1$ percent. Numbers inside parentheses are $t$-ratios.

\begin{tabular}{cccccccc}
\hline & $\rho$ & $\delta$ & $\beta$ & $\sigma_{\varepsilon}$ & $\mu_{U}^{*} / c$ & $\mu_{N}^{*} / c$ & $\mu_{B}^{*} / c$ \\
$(1)$ & $(2)$ & $(3)$ & $(4)$ & $(5)$ & $(6)$ & $(7)$ & $(8)$ \\
\hline & & & & & & & \\
AR(1) Model & 0.0548 & & & 0.00659 & 0.92 & 0.88 & 0.85 \\
& $(2.494)$ & & & & & & \\
ARMA(1,1) Model & 0.918 & 0.880 & & 0.00658 & 0.32 & 0.34 & 0.32 \\
& $(19.232)$ & $(15.665)$ & & & & & \\
MA(21) Model & & & 0.0257 & 0.00656 & 0.23 & 0.24 & 0.23 \\
& & & $(3.846)$ & & & & \\
MA(126) Model & & & 0.00818 & 0.00635 & 0.083 & 0.12 & 0.12 \\
\hline
\end{tabular}




\section{Table II Effects of Transaction Costs on Trading Performance in Foreign Exchange}

This table reports trading performance in the Japanese yen with a round-trip transaction cost $c=0.1$ percent. The data covers the period from August 31,1978 to May 3,2003 with 6,195 daily observations. The first $1 / 3$ of the sample (2,065 observations) is used for model estimation. The parameters are then used to calculate the optimal transaction cost filters. Out-of-sample forecasting starts on November 28, 1986 until the end of the sample (4,130 observations). Columns 2-6 display results where no transaction cost filter is imposed. Columns 3-4 report the before-cost returns and $t$-ratios, whereas Columbus 5-6 report the after-cost returns and $t$-ratios. Similarly, Columns 7-9 report the results (after-cost) when a naive filter equal to the actual transaction cost $c$ is imposed. Columns 10-12, 13-15, and 16-18, show the results when the optimal filters, $\mu_{U}^{*}, \mu_{N}^{*}$ and $\mu_{B}^{*}$, are imposed, respectively. All returns are annualized.

\begin{tabular}{|c|c|c|c|c|c|c|c|c|c|c|c|c|c|c|c|c|c|}
\hline & \multicolumn{5}{|c|}{ without transaction cost filter } & \multicolumn{3}{|c|}{ with naïve filter " $c$ " } & \multicolumn{3}{|c|}{ with optimal filter $\mu_{U}^{*}$} & \multicolumn{3}{|c|}{ with optimal filter $\mu_{N}^{*}$} & \multicolumn{3}{|c|}{ with optimal filter $\mu_{B}^{*}$} \\
\hline & \multirow{2}{*}{$\begin{array}{c}\# \text { of } \\
\text { Trade }\end{array}$} & \multicolumn{2}{|c|}{ before cost } & \multicolumn{2}{|c|}{ after cost } & \multirow{2}{*}{$\begin{array}{l}\text { \# of } \\
\text { trade }\end{array}$} & \multicolumn{2}{|c|}{ after cost } & \multirow{2}{*}{$\begin{array}{c}\text { \# of } \\
\text { trade }\end{array}$} & \multicolumn{2}{|c|}{ after cost } & \multirow{2}{*}{$\begin{array}{l}\text { \# of } \\
\text { trade }\end{array}$} & \multicolumn{2}{|c|}{ after cost } & \multirow{2}{*}{$\begin{array}{l}\# \text { of } \\
\text { trade }\end{array}$} & \multicolumn{2}{|c|}{ after cost } \\
\hline & & return & $t$-ratio & return & $t$-ratio & & return & $t$-ratio & & return & $t$-ratio & & return & $t$-ratio & & return & $t$-ratio \\
\hline (1) & $(2)$ & (3) & (4) & $(5)$ & (6) & $(7)$ & $(8)$ & (9) & $(10)$ & $(11)$ & $(12)$ & (13) & $(14)$ & $(15)$ & (16) & $(17)$ & $(18)$ \\
\hline \multicolumn{18}{|c|}{ Panel A. AR(1) Model } \\
\hline Long & 2100 & 0.084 & 4.097 & -0.044 & -2.151 & 34 & 0.054 & 2.199 & 42 & 0.077 & 3.507 & 48 & 0.076 & 3.458 & 66 & 0.060 & 2.855 \\
\hline Short & & 0.015 & 0.815 & 0.143 & 7.669 & & 0.045 & 3.515 & & 0.022 & 1.299 & & 0.023 & 1.373 & & 0.039 & 2.167 \\
\hline $\begin{array}{l}\text { Long- } \\
\text { Short }\end{array}$ & & 0.069 & 2.486 & -0.188 & -6.702 & & 0.009 & 0.315 & & 0.056 & 2.011 & & 0.053 & 1.910 & & 0.022 & 0.794 \\
\hline \multicolumn{18}{|c|}{ Panel B. ARMA $(1,1)$ Model } \\
\hline Long & 444 & 0.078 & 3.881 & 0.051 & 2.525 & 12 & 0.052 & 2.276 & 81 & 0.075 & 3.827 & 71 & 0.083 & 4.212 & 81 & 0.075 & 3.856 \\
\hline Short & & 0.021 & 1.110 & 0.048 & 2.533 & & 0.047 & 3.028 & & 0.024 & 1.237 & & 0.016 & 0.843 & & 0.024 & 1.208 \\
\hline $\begin{array}{l}\text { Long- } \\
\text { Short }\end{array}$ & & 0.057 & 2.054 & 0.003 & 0.093 & & 0.005 & 0.199 & & 0.051 & 1.827 & & 0.066 & 2.391 & & 0.052 & 1.870 \\
\hline \multicolumn{18}{|c|}{ Panel C. MA(21) Model } \\
\hline Long & 448 & 0.070 & 3.489 & 0.042 & 2.114 & 12 & 0.041 & 1.839 & 125 & 0.068 & 3.366 & 123 & 0.068 & 3.380 & 125 & 0.070 & 3.473 \\
\hline Short & & 0.029 & 1.536 & 0.057 & 2.952 & & 0.058 & 3.583 & & 0.031 & 1.649 & & 0.031 & 1.638 & & 0.029 & 1.543 \\
\hline $\begin{array}{l}\text { Long- } \\
\text { Short }\end{array}$ & & 0.040 & 1.453 & -0.015 & -0.522 & & -0.016 & -0.586 & & 0.036 & 1.314 & & 0.037 & 1.323 & & 0.040 & 1.451 \\
\hline \multicolumn{18}{|c|}{ Panel D. MA(126) Model } \\
\hline Long & 131 & 0.082 & 4.123 & 0.074 & 3.717 & 5 & 0.039 & 1.816 & 31 & 0.078 & 3.934 & 29 & 0.072 & 3.631 & 29 & 0.072 & 3.631 \\
\hline Short & & 0.017 & 0.893 & 0.025 & 1.307 & & 0.060 & 3.481 & & 0.021 & 1.083 & & 0.027 & 1.391 & & 0.027 & 1.391 \\
\hline Long- & & & & & & & & & & & & & & & & & \\
\hline Short & & 0.065 & 2.338 & 0.049 & 1.756 & & -0.020 & -0.725 & & 0.057 & 2.074 & & 0.046 & 1.650 & & 0.046 & 1.650 \\
\hline
\end{tabular}




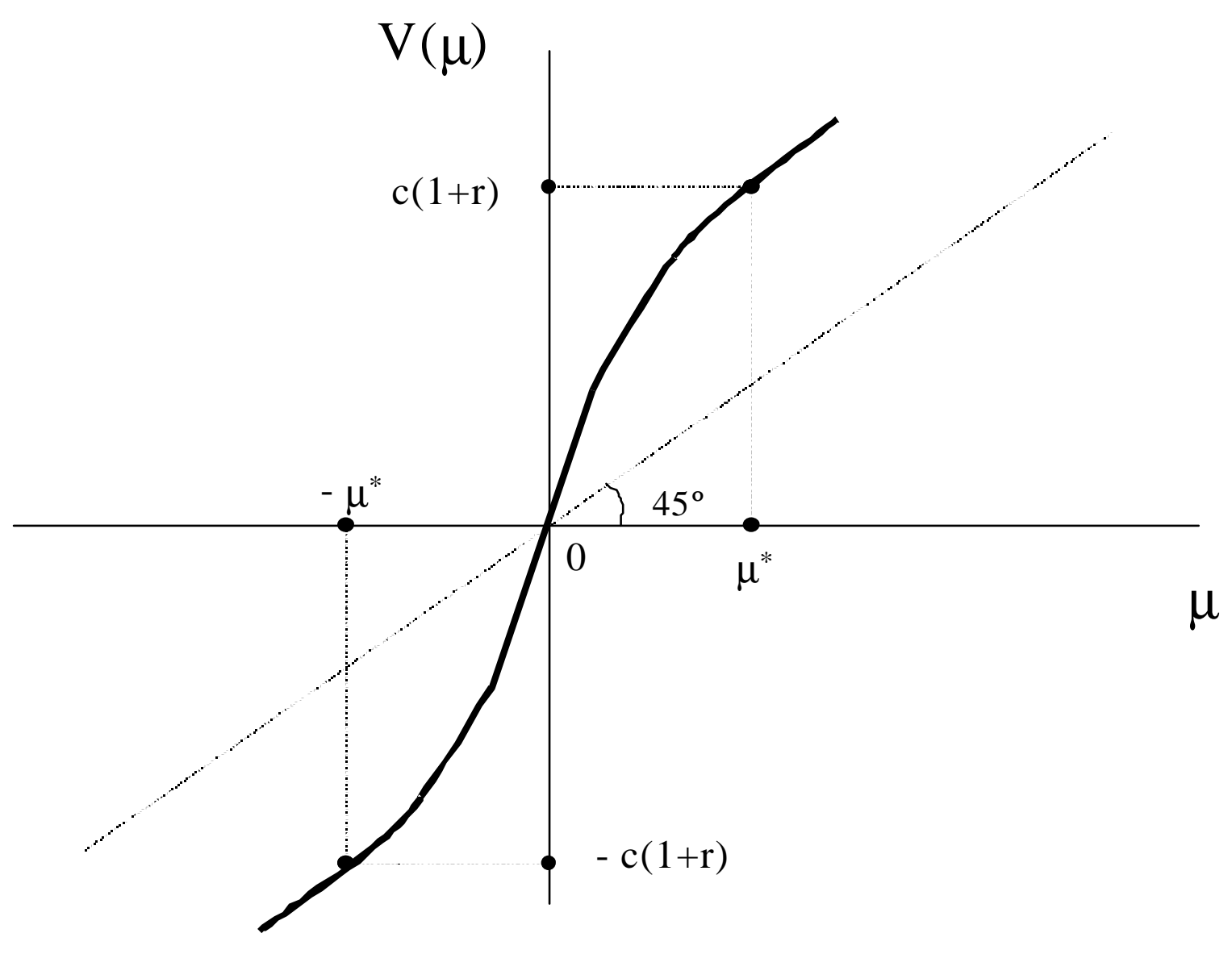

Figure 1: The Excess Value Function 
Fig 2. After-cost Excess Return (round) and Trading Cost (square) vs. $\mu / c$,

Vertical Lines: Solid $\mu_{\mathrm{U}}^{*} / \mathrm{c}$, Dash $\mu_{\mathrm{N}}^{*} / \mathrm{c}$, Long Dash $\mu_{\mathrm{B}}^{*} / \mathrm{c}$ $c=0.1 \%, A R(1)$ Model

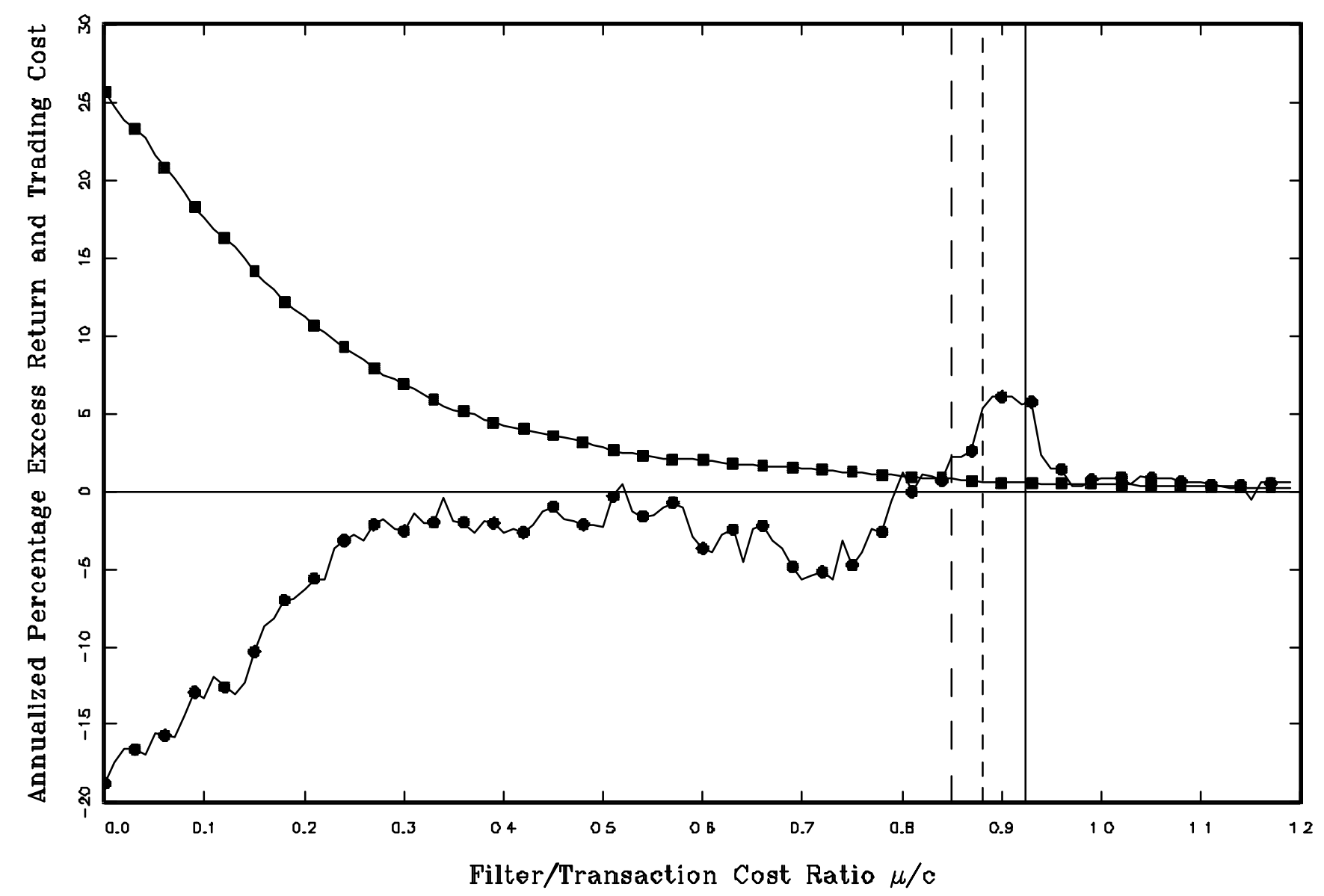


Fig 3. After-cost Excess Return (round) and Trading Cost (square) vs. $\mu / c$,

Vertical Lines: Solid $\mu_{\mathrm{U}}^{*} / \mathrm{c}$, Dash $\mu_{\mathrm{N}}^{*} / \mathrm{c}$, Long Dash $\mu_{\mathrm{B}}^{*} / \mathrm{c}$ $c=0.1 \%, \operatorname{ARMA}(1,1)$ Model

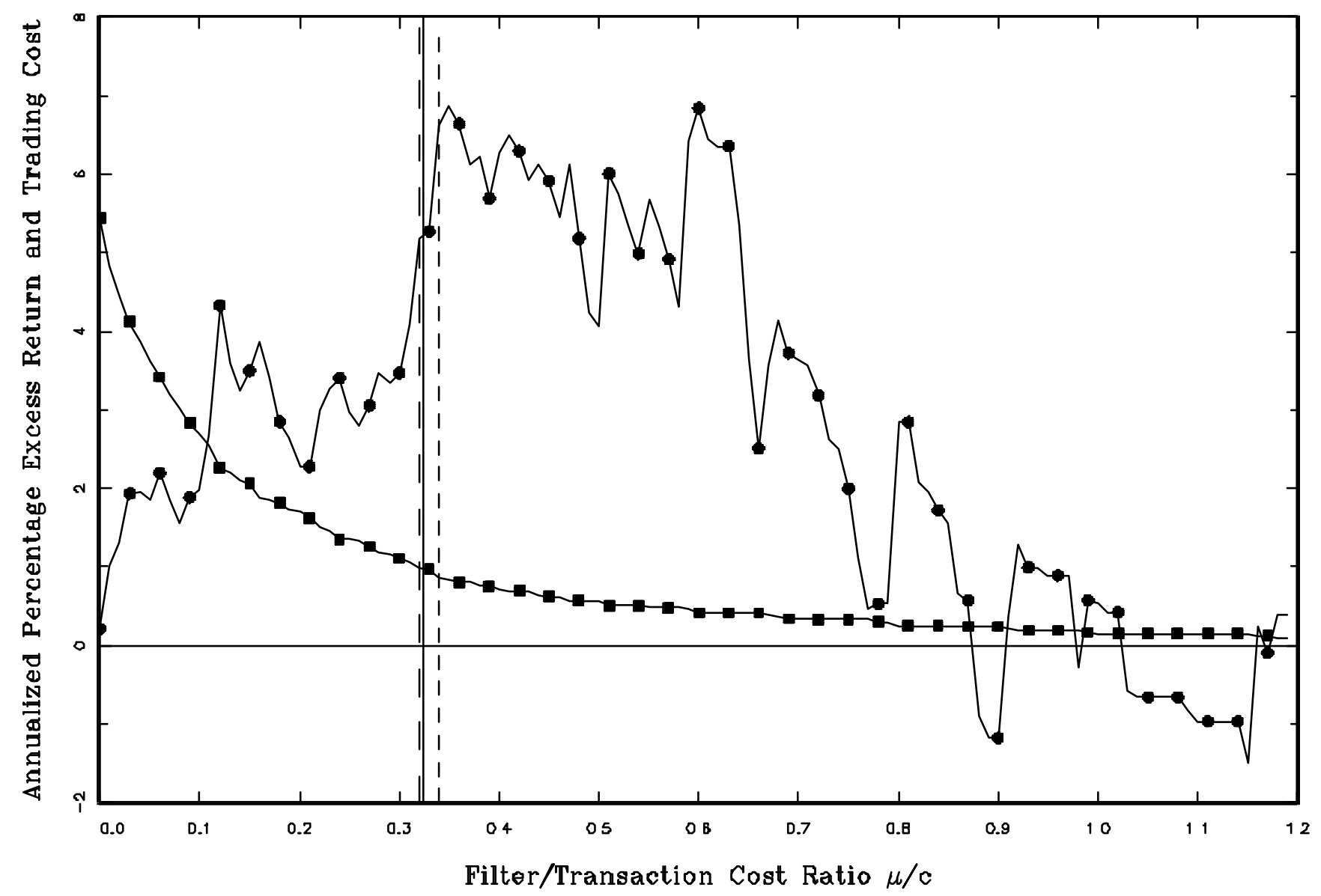


Fig 4. After-cost Excess Return (round) and Trading Cost (square) vs. $\mu / c$, Vertical Lines: Solid $\mu_{\mathrm{U}}^{*} / c$, Dash $\mu_{\mathrm{N}}^{*} / c$, Long Dash $\mu_{\mathrm{B}}^{*} / c$ $c=0.1 \%$, MA(21) Model

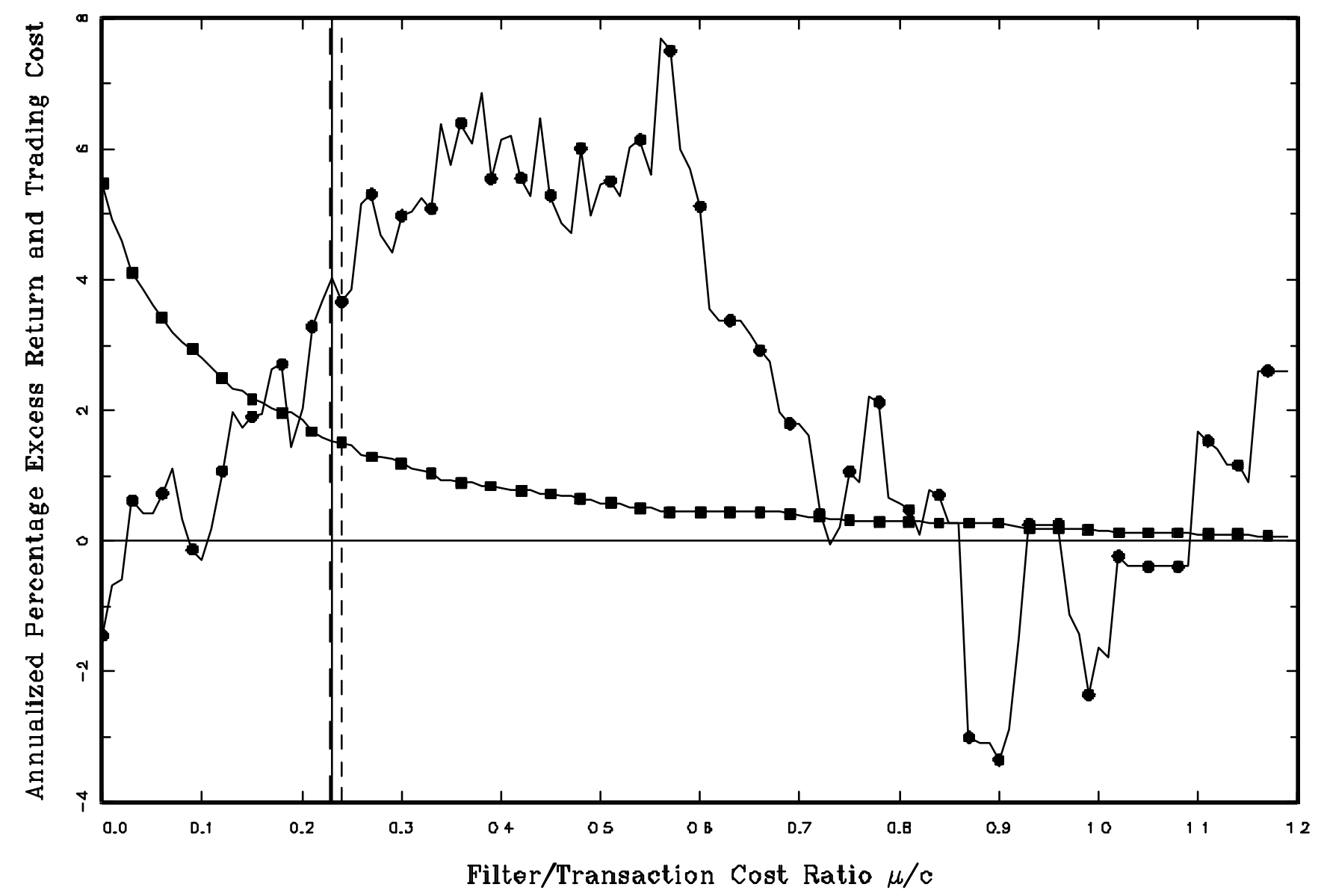


Fig 5. After-cost Excess Return (round) and Trading Cost (square) vs. $\mu / c$, Vertical Lines: Solid $\mu_{\mathrm{U}}^{*} / c$. Dash $\mu_{\mathrm{N}}^{*} / c$, Long Dash $\mu_{\mathrm{B}}^{*} / c$ $c=0.1 \%, \operatorname{MA}(126)$ Model

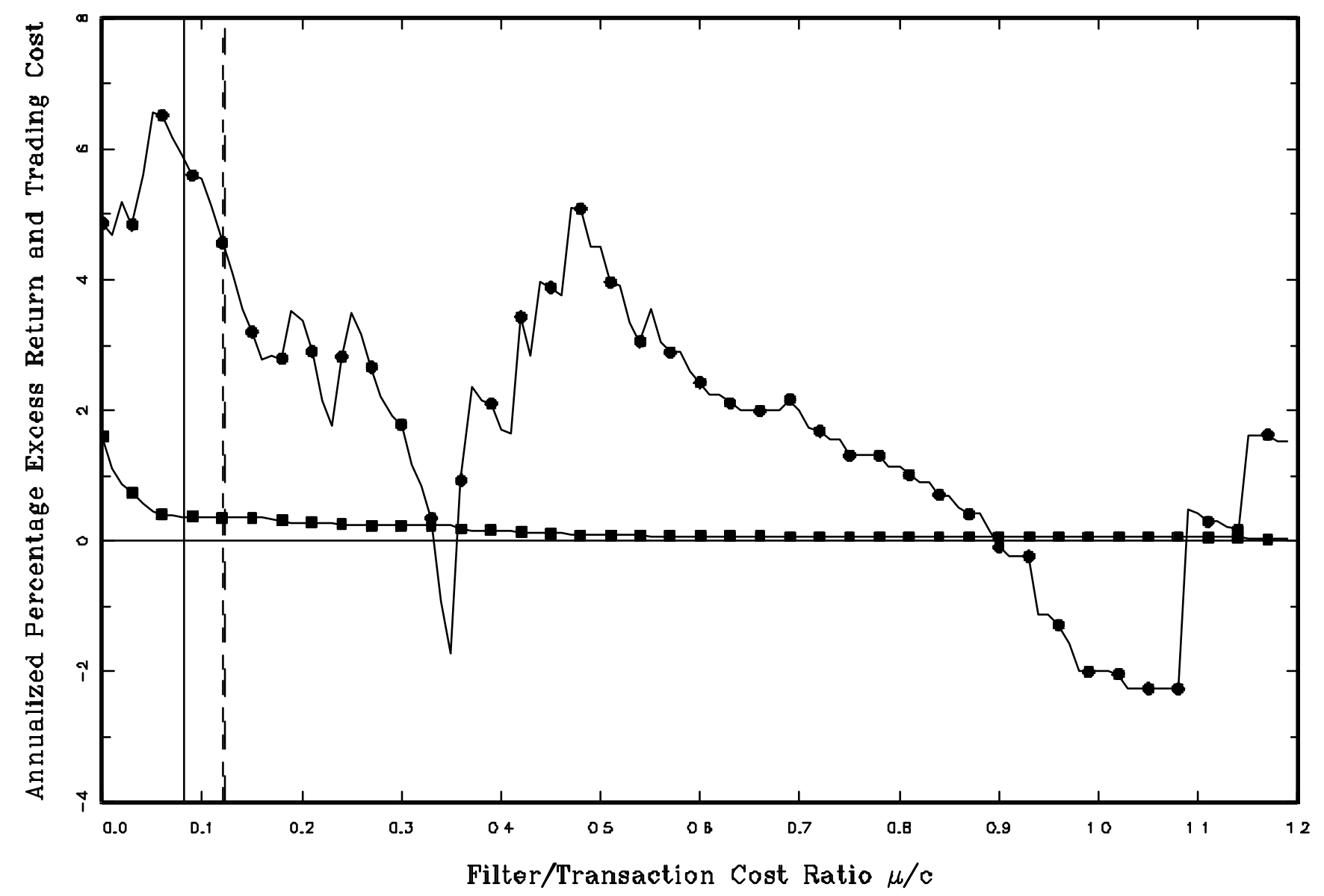

